\title{
Compensation between Resolved and Unresolved Wave Drag in the Stratospheric Final Warmings of the Southern Hemisphere
}

\author{
Guillermo SCHEFFLER AND MANUEl Pulido \\ Department of Physics, Facultad de Ciencias Exactas y Naturales y Agrimensura, Universidad Nacional del Nordeste, and IMIT, \\ UMI-IFAECI/CNRS, CONICET, Corrientes, Argentina
}

(Manuscript received 18 September 2014, in final form 23 June 2015)

\begin{abstract}
The role of planetary wave drag and gravity wave drag in the breakdown of the stratospheric polar vortex and its associated final warming in the Southern Hemisphere is examined using reanalyses from MERRA and a middle-atmosphere dynamical model. The focus of this work is on identifying the causes of the delay in the final breakdown of the stratospheric polar vortex found in current general circulation models. Sensitivity experiments were conducted by changing the launched momentum flux in the gravity wave drag parameterization. Increasing the launched momentum flux produces a delay of the final warming date with respect to the control integration of more than 2 weeks. The sensitivity experiments show significant interactions between planetary waves and unresolved gravity waves. The increase of gravity wave drag in the model is compensated by a strong decrease of Eliassen-Palm flux divergence (i.e., planetary wave drag). This concomitant decrease of planetary wave drag is at least partially responsible for the delay of the final warming in the model. Experiments that change the resolved planetary wave activity entering the stratosphere through artificially changing the bottom boundary flux of the model also show an interaction mechanism. Gravity wave drag responds via critical-level filtering to planetary wave drag perturbations by partially compensating them. Therefore, there is a feedback cycle that leads to a partial compensation between gravity wave and planetary wave drag.
\end{abstract}

\section{Introduction}

The stratosphere at high latitudes exhibits an annual cycle that is dominated by the evolution of the stratospheric polar vortex. This vortex reaches its maximum intensity during winter with strong circumpolar westerlies. Then, during spring, the westerlies slowly weaken and turn to easterlies in the mid- and upper stratosphere, while mild westerlies may remain in the lower stratosphere. This polar vortex breakdown is produced by what is called final warming. In this work, the day of the transition from westerly to easterly wind at $60^{\circ} \mathrm{S}$ and $10 \mathrm{hPa}$ is what we refer to as the final warming date.

General circulation models and chemistry-climate models show a pronounced bias in the representation of the processes related to the vortex breakdown. This bias, commonly known as cold-pole bias, is characterized by lower temperatures in winter in the polar regions

Corresponding author address: Manuel Pulido, Facultad de Ciencias Exactas, Universidad Nacional del Nordeste, Av. Libertad 5400, Corrientes 3400, Argentina.

E-mail: pulido@unne.edu.ar and a stronger-than-observed polar vortex, which then breaks down too late. Eyring et al. (2006) showed that the transition from westerlies to easterlies at $60^{\circ} \mathrm{S}$ occurs too late in most of the current coupled chemistryclimate models, while in one of the models this transition does not occur at all. In a more recent chemistryclimate model intercomparison, Butchart et al. (2011) analyzed 16 models and showed different metrics to assess their performance with respect to several key processes of stratospheric dynamics. A wide spread of model performance was found among the different models. The different diagnostics show a consistent poorer performance of the models in the Southern Hemisphere. The worst diagnostic metrics corresponded to the delay of the final warming date and a toocold springtime polar cap temperature in the Southern Hemisphere.

Black and McDaniel (2007) showed that stratospheric final warmings-defined by them as the last day in which the zonal mean zonal wind at $60^{\circ} \mathrm{S}$ and $50 \mathrm{hPa}$ drops below $10 \mathrm{~m} \mathrm{~s}^{-1}$-have a significant impact in the largescale circulation, both in the stratosphere and the troposphere. Stratospheric final warming events introduce 
an important source of interannual variability, which links the stratosphere to the troposphere. These results highlight the need for a precise representation of the final warming in general circulation models. The tropospheric variability found in response to the stratospheric polar vortex variability shown in Black and McDaniel (2007) for stratospheric final warming events differs from the usual response, in which the stratospheretroposphere coupling is manifested in the tropospheric annular modes (e.g., Baldwin et al. 2003; Gerber et al. 2010; Simpson et al. 2011). An accurate representation of the time of breakdown is also critical in the estimation of trends in Antarctic ozone transport, which has been shown to be sensitive to changes in the stratospheric mean circulation (Stolarski et al. 2006).

The presence of the bias in the evolution of the polar vortex breakdown on most general circulation models is associated with a poor representation of wave drag in the stratosphere, though it is not clear whether the reason for the bias is a bad representation of gravity wave drag given by the parameterizations of unresolved gravity waves in the model or an incorrect or insufficient amount of planetary wave drag, which is resolved directly in the model.

McLandress et al. (2012) used wind increments from the Canadian Middle Atmosphere Model Data Assimilation System (CMAM-DAS) to infer the systematic bias in the model. The largest systematic biases are found around $60^{\circ} \mathrm{S}$ during winter. These systematic biases are interpreted as missing wave drag in the model. They were able to reproduce this missing wave drag in a free model integration by adding an artificial topography at $60^{\circ} \mathrm{S}$ in the orographic gravity wave drag parameterization. McLandress et al. (2012) showed that additional orographic gravity wave drag at $60^{\circ} \mathrm{S}$ leads to a reduction in the zonal mean zonal wind and temperature biases in the Southern Hemisphere winter, along with an improvement in the time of breakdown of the stratospheric polar vortex. Previous studies have shown that the incorporation of nonorographic gravity wave parameterizations produces an important reduction of the cold-pole bias (e.g., Manzini and McFarlane 1998). Along these lines, Austin et al. (2003) also focused on the impact of nonorographic gravity wave drag schemes; they showed in a comparison of several chemistry-climate models and observations that polar temperature biases in the middle stratosphere in the Southern Hemisphere winter and spring were smaller in those models that incorporated a nonorographic gravity wave drag scheme [e.g., the Unified Model with Eulerian Transport and Chemistry (UMETRAC) and CMAM], compared with models that do not represent nonorographic gravity wave drag.
An inaccurate representation of the processes that generate large-scale waves may also impact the vortex breakdown bias found in general circulation models. Austin et al. (2003) also found that, during Southern Hemisphere winter, models with lower horizontal resolution show a weaker response in temperature to changes in the heat flux. This is attributed mostly to the inability of low-resolution models to capture the highamplitude planetary wave events. Hurwitz et al. (2010) showed that the delay in the timing of the zonal mean wind transitions to easterlies at $10 \mathrm{hPa}$ in the Goddard Earth Observing System Chemistry-Climate Model (GEOSCCM) is related to a lack of heat flux at $100 \mathrm{hPa}$ during October and November. In addition to these results with GEOSCCM, they show that the United Kingdom Chemistry and Aerosol model (UKCA), NCEP-2, and ERA-40 have a strong correlation between heat fluxes at $100 \mathrm{hPa}$ and the timing of polar vortex breakdown: that is, weaker heat fluxes than the mean observed heat flux in midlatitudes during October and November are related to a delayed transition to easterlies in the stratosphere. Additionally, Garfinkel et al. (2013) showed that changes in the parameterizations that have an impact on the mechanisms of planetary wave generation, in particular when updating the GEOSCCM air-sea roughness parameterization, produce some reduction in the final warming date bias in the Southern Hemisphere. The improvement is related to an enhanced upward wave activity flux entering the stratosphere in September and October, explained by a wave-1 pattern in the zonal wind produced by the zonally asymmetric response of eddy fluxes to the enhanced roughness.

Identifying which type of wave drag has the dominant role in the appearance of the model bias in final warmings has an additional complexity, given that perturbations to either resolved or unresolved waves tend to partially compensate each other. McLandress et al. (2012) showed that the incorporation of extra gravity wave drag around $60^{\circ} \mathrm{S}$ would lead to a weakening and latitudinal spreading of planetary wave drag. This effect was explained by deterioration in the conditions for vertical propagation as a result of a weakening of zonal winds and of a weakening of the meridional gradient of potential vorticity.

Three different mechanisms were proposed by Cohen et al. (2014) to explain the interactions between resolved and unresolved wave drag. The mechanisms are associated with stability constraints (discussed in Cohen et al. 2013), potential vorticity mixing constraints, and resolved and unresolved wave drag interactions through planetary wave refractive index changes by the gravity wave drag. Cohen et al. (2014) showed that these mechanisms, while not mutually exclusive, depend on the location of the gravity wave drag with respect to the surf zone. Sigmond 
and Shepherd (2014) examined the interaction between nonlocalized perturbations of orographic gravity wave drag and resolved wave drag and their contribution to the Brewer-Dobson circulation in the context of climate change. The impact of an increase of orographic gravity wave drag in the Brewer-Dobson circulation is largely compensated by a decrease of planetary wave drag. While the compensation mechanism does not hold for Northern high latitudes, it is present for Southern high latitudes and occurs for both current climate and future climate scenarios.

We examine the relation between stratospheric final warming date and wave drag employing a middleatmosphere model with several model configurations to represent scenarios with different planetary wave drag and unresolved gravity wave drag. A description of the middle-atmosphere model we use is given in section 2. In the results, we compare the total unresolved wave drag from NASA's Modern-Era Retrospective Analysis for Research and Applications (MERRA) to the one obtained with the nonorographic parameterization in the model integrations (section 3). Parameterized gravity wave and resolved wave drag from the free model integration are compared with the ones from model experiments with increased and decreased planetary wave drag and nonorographic wave drag. Significant interactions are detected between both types of wave drag (sections 3a and 3c), and a possible explanation is given for the response in each type of drag in sections $3 \mathrm{~b}$ and $3 \mathrm{~d}$. Finally, some conclusions are drawn in section 4.

\section{Data and methods}

In this work, MERRA results, available data from NASA's Global Modeling and Assimilation Office (Global Modeling and Assimilation Office 2011), are examined from year 2003 to 2009 ( $7 \mathrm{yr}$ ). This reanalysis has a horizontal resolution of $1.25^{\circ} \times 1.25^{\circ}$ and 42 vertical levels, spanning up to $0.1 \mathrm{hPa}$. The MERRA system is based on the GEOS-5 atmospheric general circulation model, which includes both an orographic and a nonorographic gravity wave drag parameterization (Rienecker et al. 2011). The total forcing from the gravity wave drag parameterizations is available in the MERRA archive. The MERRA assimilation system employs a three-dimensional variational data assimilation (3DVAR) algorithm and incorporates analysis innovations through the incremental analysis update (IAU) approach (Bloom et al. 1996). The analysis correction within the IAU approach is applied through forcing terms so that the integration evolves smoothly between the assimilation windows.

MERRA results are compared in this work with integrations using the University of Reading middleatmosphere dynamical model. This model represents the full hydrostatic dynamical equations on a hexagonalicosahedral horizontal grid with 16 isentropic vertical levels (Thuburn 1997; Pulido and Thuburn 2005). It has a horizontal resolution of about $4^{\circ}$. The model height range is from about 100 to $0.01 \mathrm{hPa}$. The bottom boundary of the model at $100 \mathrm{hPa}$ is forced every $6 \mathrm{~h}$ with the Montgomery potential taken from MERRA so that a realistic representation of the tropospheric large-scale disturbances is forcing the bottom of the model (contrary to atmospheric general circulation models, which may have biases in the representation of planetary waves entering the stratosphere). The model contains a Rayleigh sponge layer on the top to avoid wave reflection. It also includes a radiative transfer scheme representing solar heating and the effects of $\mathrm{CO}_{2}, \mathrm{O}_{3}$, and $\mathrm{H}_{2} \mathrm{O}$ (Shine 1987). Monthly mean vertical profiles of $\mathrm{O}_{3}$ mixing ratio are used, and they are kept invariant along the model integration, while the $\mathrm{CO}_{2}$ mixing ratio is also considered invariant. The gravity wave drag parameterization implemented in the model is the one introduced in Scinocca (2003). This is a nonhydrostatic nonrotational nonorographic spectral gravity wave drag parameterization. This scheme represents a timeindependent and horizontally uniform spectrum that is launched at $100 \mathrm{hPa}$ and propagates upward, undergoing processes of back-reflection and critical-level filtering.

Since we focused on the Southern Hemisphere vortex breakdown, we conducted independent model integrations for each year, taking initial conditions on 1 January each year from MERRA. Taking initial conditions every 1 January eliminates the model systematic bias from the previous year. The model bias is expected to include all the time scales that contribute to atmospheric variability. Even though the seasonal time scale may be dominant in the model bias, an interannual time scale may be also present; therefore, taking initial conditions every 1 January eliminates this component of the model bias. In this way, we focus on the differences in the seasonal cycle between the model and reanalysis. Under these conditions, we integrated the model for 7 years (2003-09). The integration of the model with standard configuration will be referred to as control integration.

As our aim is to understand the role of planetary wave drag versus gravity wave drag in determining stratospheric final warmings, we conducted two sets of free model integrations.

The first set of experiments is focused on the sensitivity of the model to the strength of gravity wave drag given by the parameterization. The only parameter that we varied from Scinocca's parameterization is the total integrated gravity wave momentum flux at launch height, denoted by $\rho_{0} F_{p}^{\text {total }}$ in Scinocca (2003); here, we refer to this parameter as launched momentum flux parameter. This represents the amplitude of the gravity wave momentum 
flux (spectrally integrated in vertical wavenumber and intrinsic frequency) in each azimuthal direction. The launched spectrum of waves is assumed to be isotropic. The rest of the tunable parameters are kept in the standard values, which are the ones suggested in Scinocca (2003) and used in other general circulation models (e.g., CMAM). The reference value we used for the launched momentum flux parameter is $25 \sqrt{2} \times 10^{-4} \mathrm{~Pa}$, this is the optimal value obtained in Pulido et al. (2012) for high latitudes in the Southern Hemisphere winter. Note that this value is 10 times greater than the standard value suggested in Scinocca (2003). For the strong gravity wave drag experiment, we increase the mentioned parameter by a factor of 2 from the reference value. For the weak gravity wave drag experiment, we decrease the launched momentum flux parameter by a factor of 0.1 [so the weak gravity wave drag experiment corresponds to the standard parameter value suggested in Scinocca (2003)].

The second set of experiments examines the sensitivity of stratospheric final warmings simulated by the model to planetary wave drag. Since the model we use is a middle-atmosphere dynamical model, it allows us to readily change the large-scale wave activity entering the model from the troposphere. We conducted one integration of the model in which the anomalies of the Montgomery potential at the bottom boundary of the model taken from MERRA were amplified by $25 \%$ (initially, we tried with a $50 \%$ amplification, but the increased wave activity produced instabilities during the integration of the model). The second integration within this set of experiments corresponds to a reduction of $50 \%$ of the Montgomery potential anomalies at $100 \mathrm{hPa}$. As will be explained in section 3a, the delay of the final warming in our model may not be attributable to an inaccurate Eliassen-Palm flux entering from the troposphere. However, with this set of experiments, we expect to address the interaction mechanism between planetary wave drag changes and the gravity wave drag responses in the context of stratospheric final warmings.

Since the dynamical model has isentropic coordinates as vertical levels, the meridional and vertical components of the resolved Eliassen-Palm flux for the model are expressed in these coordinates (Andrews et al. 1987):

$$
\begin{aligned}
& F_{\phi}=-a \cos \phi \overline{(\sigma v)^{\prime} u^{\prime}}, \\
& F_{\theta}=g^{-1} \overline{p^{\prime} M^{\prime}}-a \cos \phi \overline{(\sigma Q)^{\prime} u^{\prime}},
\end{aligned}
$$

where $a, u, v, p, M, Q$, and $\sigma$ are, respectively, Earth's radius, zonal and meridional wind components, atmospheric

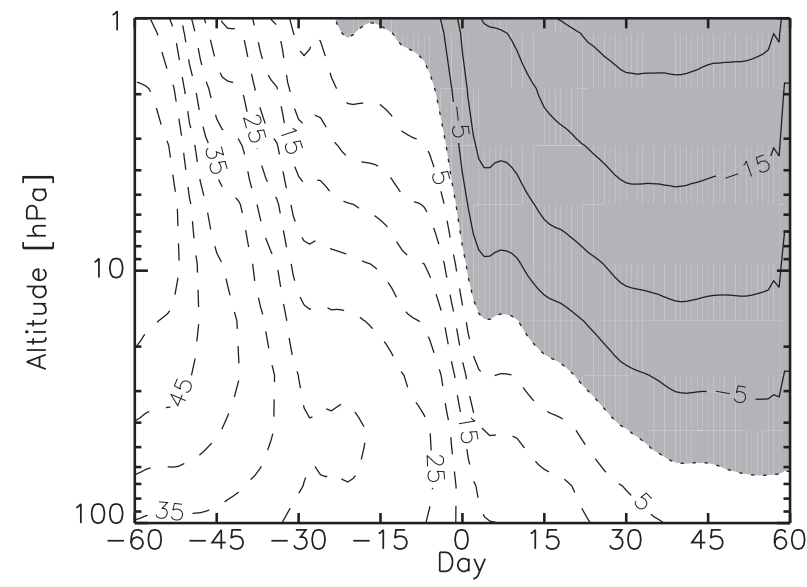

FIG. 1. Composite of zonal mean zonal wind with respect to stratospheric final warming date in MERRA for 2003-09 averaged between $80^{\circ}$ and $50^{\circ} \mathrm{S}$. Contour interval is $5 \mathrm{~m} \mathrm{~s}^{-1}$; negative values (westward winds) are shaded.

pressure, Montgomery's potential, potential vorticity, and air density (in isentropic coordinates). Overlines represent the zonal mean, and primes represent anomalies to the zonal mean.

The resolved wave drag is given by the divergence of the Eliassen-Palm flux (EPFD):

$$
\nabla \cdot \mathbf{F}=(a \cos \phi)^{-1} \frac{\partial}{\partial \phi}\left(F_{\phi} \cos \phi\right)+\frac{\partial F_{\theta}}{\partial \theta},
$$

where $\theta$ is the potential temperature which is used as vertical coordinate.

\section{Results}

Figure 1 shows the 7 -yr composite of zonal mean zonal wind from MERRA during the stratospheric final warming, averaged between $80^{\circ}$ and $50^{\circ} \mathrm{S}$. The composite was constructed with respect to the date when the zonal mean zonal wind reverses from westerlies to easterlies at $60^{\circ} \mathrm{S}$ and $10 \mathrm{hPa}$ and remains easterly until the next autumn. The term "final warming date" without an explicit height reference will refer to the date when this criterion is accomplished at $10 \mathrm{hPa}$. The final warming date ranges between the first week of November and the first week of December, with a mean final warming date on 16 November and a standard deviation of 11.2 days. As expected, Fig. 1 shows the reversal of the zonal wind in the Southern Hemisphere during the stratospheric final warming starting in the upper stratosphere and descending to the lower stratosphere as time goes by, while a weakening of the eastward zonal wind is found in the lowest part of the stratosphere. Two descending rates are found in the zero 


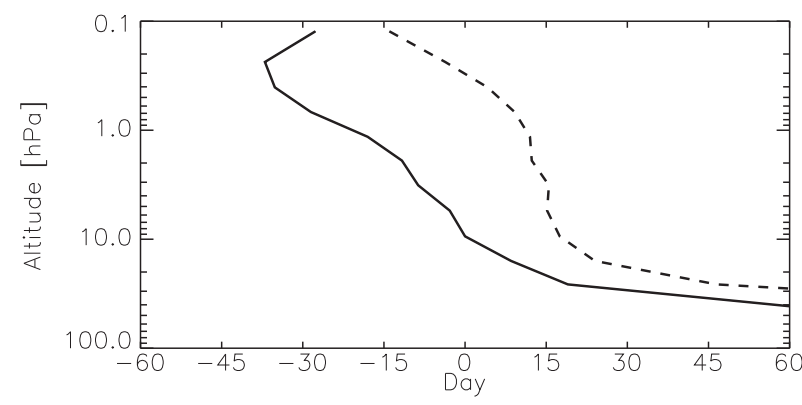

FIG. 2. Descent of the zero zonal mean zonal wind line at $60^{\circ} \mathrm{S}$ for MERRA (continuous line) and for control integration (dashed line) using MERRA as a reference for the composites.

zonal wind line of MERRA, one with steep tilt in the upper stratosphere (above $15 \mathrm{hPa}$ ), and the other with a gentler tilt in the lower stratosphere (below $15 \mathrm{hPa}$ ).

Figure 2 shows the transition from westerlies to easterlies at $60^{\circ} \mathrm{S}$ for MERRA and the control integration. Both composites were taken with respect to the final warming date in MERRA. Note that the descent of the zero zonal mean wind is shown at $60^{\circ} \mathrm{S}$ latitude and up to a height of $0.1 \mathrm{hPa}$, because this is used as the standard diagnostic (Eyring et al. 2006; Butchart et al. 2011). For the budget analyses, a high-latitude average (i.e., $50^{\circ}-$ $80^{\circ} \mathrm{S}$ average) and up to $1 \mathrm{hPa}$ are shown. The control integration (dashed line in Fig. 2) shows a delay in the final warming of 16 days at $10 \mathrm{hPa}$ and of 31 days at $1 \mathrm{hPa}$. The delay found in the control integration in the lower stratosphere up to $10 \mathrm{hPa}$ is similar to the one found in other general circulation models (e.g., Eyring et al. 2006; Butchart et al. 2011). The standard deviation at $10 \mathrm{hPa}$ in the control experiment is 13.5 days. At this height, the control integration exhibits a slight higher variability in the final warming date than the one in MERRA. This difference in the standard deviation is not statistically significant, considering the uncertainties in the estimates and the small number of events $(7 \mathrm{yr})$. Apart from the delay in the wind reversal, the control model integration produces a sudden and rapid wind reversal between 0.4 and $15 \mathrm{hPa}$, contrary to the slowerpaced final warming found in MERRA at $60^{\circ} \mathrm{S}$. The descent line at $60^{\circ} \mathrm{S}$ found in the model integration looks more similar in terms of the sudden wind reversal to the $50^{\circ}-80^{\circ} \mathrm{S}$ average from MERRA shown in Fig. 1.

One possible candidate for the delay in the model is an inadequate representation of planetary wave generation. It should be noticed that the bottom of our model is located at the tropopause, so the wave activity entering the stratosphere is realistic and imposed entirely by realistic bottom boundary conditions taken from MERRA, unlike general circulation models that propagate waves from the surface and rely on the quality of their (tropospheric) parameterizations to represent precisely planetary wave generation. Since the inadequate representation of planetary waves entering the stratosphere is discarded as being responsible for the delay in the final warming found in the control integration. The other two possible (related) candidates for the delay are that planetary waves do not break at the correct location because of biases in the mean winds and that there is a deficient representation of the forcing produced by small-scale processes not resolved by the model. The planetary wave propagation in models is affected by the unresolved gravity wave drag through mean wind changes, so that the breaking of planetary waves will be not correct if the gravity wave drag is not well represented.

Figure 3a shows the zonal mean zonal gravity wave drag provided by the gravity wave parameterizations of the MERRA model (GEOS-5) for the latitudinal band of $80^{\circ}-50^{\circ} \mathrm{S}$. The parameterized gravity wave drag in GEOS-5 is produced by a nonorographic and an orographic gravity wave parameterization (Rienecker et al. 2011). The gravity wave drag is mainly negative (westward acceleration) during the examined period. The minimum of zonal missing forcing $\left(-5.4 \mathrm{~m} \mathrm{~s}^{-1}\right.$ day $\left.^{-1}\right)$ occurs at $1 \mathrm{hPa}$, and it happens 39 days before the final warming. Except for the descent of the zero zonal wind line, we constrain our analysis below $1 \mathrm{hPa}$ to avoid back-reflection effects and effects of the sponge layer close to the top of the model and close to the top of observations (in MERRA). MERRA increments are shown in Fig. 3b. These increments may be thought of as the missing forcing of GEOS-5, which, together with the other parameterized forcings, constitutes the total momentum forcing on the model. According to these increments, around 2 months before the final warming, the GEOS-5 gravity wave drag parameterizations do not produce enough deceleration on the mean flow in high latitudes; however, gravity wave drag deceleration is too strong close to and after the wind reversal.

Zonal mean gravity wave drag from the parameterization in the control integration (Fig. 3c) between 60 and 40 days prior to the final warming date shows westward forcing above about $30 \mathrm{hPa}$. This is in accordance to MERRA gravity wave drag parameterizations and increments, where the largest deceleration forcing occurs during the vortex breakdown, more than one month before the final warming. After day -40 , the westward acceleration descends with the jet, and an eastward acceleration is established above $20 \mathrm{hPa}$. On the other hand, MERRA gives westward acceleration there. Therefore, during the transition from westerly to easterly wind, the nonorographic parameterization gives a forcing that is against the transition. This suggests that 

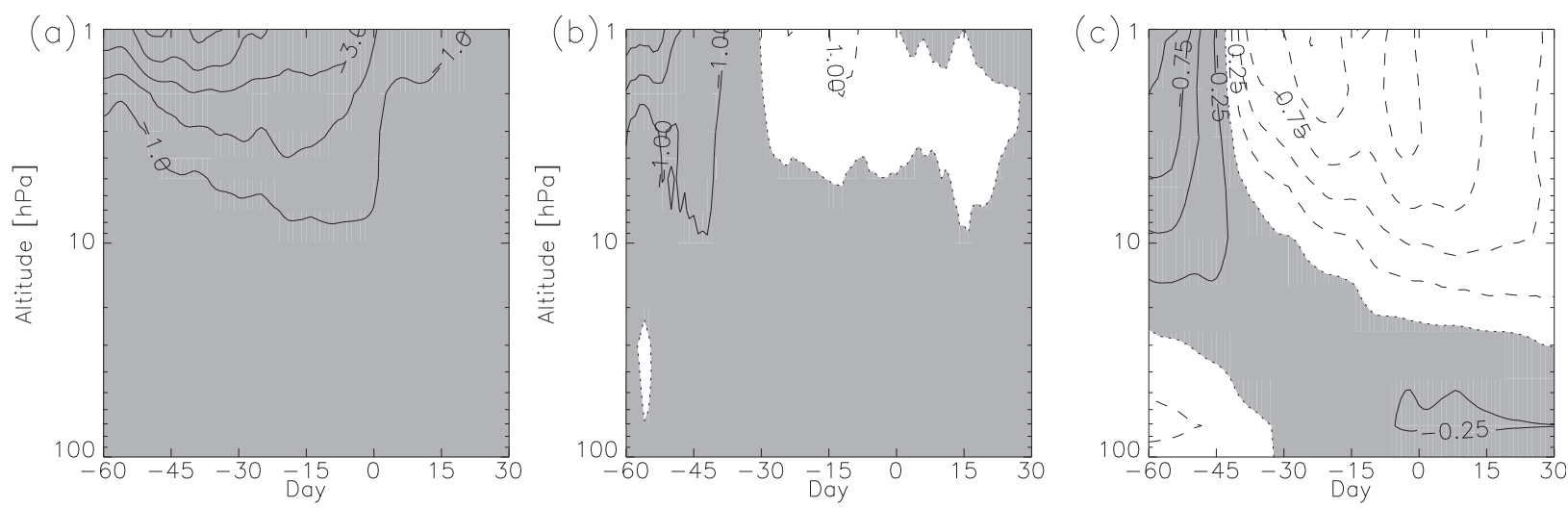

FIG. 3. (a) Zonal mean zonal forcing averaged between $80^{\circ}$ and $50^{\circ} \mathrm{S}$ as a function of time from MERRA orographic and nonorographic gravity wave drag parameterizations. (b) Zonal mean zonal increments from MERRA assimilation. Contour intervals are $1 \mathrm{~m} \mathrm{~s}^{-1}$ day ${ }^{-1}$. (c) Zonal mean zonal forcing from the nonorographic gravity wave drag parameterization in the control integration. Contour interval is $0.25 \mathrm{~m} \mathrm{~s}^{-1} \mathrm{day}^{-1}$. Positive values are shown with dashed contour lines. Negative values are shaded and shown with continuous contour lines.

the nonorographic gravity wave parameterization does not give the correct forcing for those dates or that the orographic gravity wave parameterization, not present in the model, may play a dominant role in the period between day 45 and the final warming date. The process that produces the change of sign in the gravity wave drag given by the parameterization is explained in the appendix.

Eliassen-Palm flux divergence derived from MERRA in the latitudinal band from $80^{\circ}$ to $50^{\circ} \mathrm{S}$ (Fig. 4a) shows several intermittent peaks of planetary wave activity during the vortex breakdown. The largest peak at $1 \mathrm{hPa}$ of $-10.4 \mathrm{~m} \mathrm{~s}^{-1}$ day $^{-1}$ coincides with the wind reversal at that height, 36 days before the final warming date (at $10 \mathrm{hPa}$ ). EPFD weakens at $1-4 \mathrm{hPa}$ during the 15 days prior to the final warming, when the jet is already weak. During the week of the final warming, there is a strong zonal deceleration EPFD peak centered around $15 \mathrm{hPa}$. This peak is associated with the change of sign in the zonal wind.

The control integration (Fig. 4b) shows three main peaks of EPFD during the analyzed period. There are two deceleration peaks at 50 and 40 days before the final warming date, reaching up to 5 and $6.25 \mathrm{~m} \mathrm{~s}^{-1}$ day $^{-1}$, respectively, at $1 \mathrm{hPa}$. Both peaks are weaker than in MERRA. The third peak, centered at $9 \mathrm{hPa}$ occurs around 11 days before the final warming. This deceleration is also smaller than the deceleration that occurred during the final warming week in MERRA.

\section{a. Dependence of the stratospheric final warming on the strength of the parameterized gravity wave drag}

We conducted sensitivity experiments by changing the magnitude of the launched gravity wave momentum flux. We performed one integration doubling the launched gravity wave momentum flux from the one used in the control integration and another with a launched gravity wave momentum flux 10 times smaller than the reference value. Figure 5 shows the date when the zonal mean zonal wind at $60^{\circ} \mathrm{S}$ drops below zero for the two experiments compared to the control experiment. The composite in the three model integrations is taken with respect to the final warming dates of the control integration in Fig. 5 to focus on the sensitivity with respect to the control integration. Note that the reference final warming dates for the composites in Fig. 2 were taken from MERRA.

Increasing the launched momentum flux in the parameterization is detrimental to an accurate representation of the stratospheric final warming, as shown in Fig. 5. Above $1 \mathrm{hPa}$, the experiment with increased launched momentum flux has between 3 and 10 days of delay with respect to the control integration (41 days of delay at $1 \mathrm{hPa}$ with respect to MERRA). The delay in the wind reversal grows when approaching the middle stratosphere and the wind reversal does not take place below $9 \mathrm{hPa}$. Two factors contribute to this counterintuitive delay: The stronger gravity wave drag produces, through zonal wind changes, a weaker Eliassen-Palm flux divergence. Furthermore, the sign of the gravity wave drag is inverted before the mean zonal wind changes of sign, acting against the westerly-to-easterly wind transition. As shown in the appendix, the change of sign in gravity wave drag in the parameterization is governed by the change in zonal mean zonal wind shear instead of changes of zonal wind sign.

The experiment with weak launched gravity wave momentum flux (and, therefore, weak gravity wave 
(a)

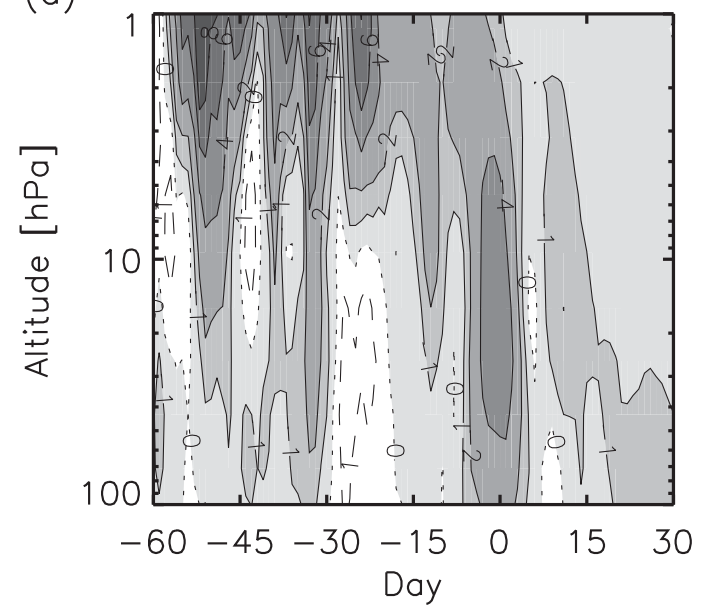

(b)

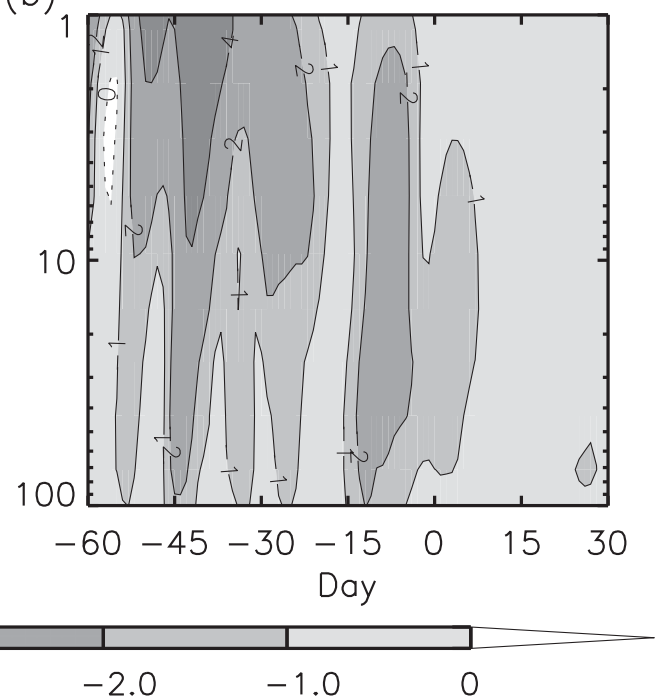

$-8.0 \quad-6.0 \quad-4.0$

FIG. 4. Eliassen-Palm flux divergence $\left(\mathrm{m} \mathrm{s}^{-1} \mathrm{day}^{-1}\right)$ as a function of time averaged between $80^{\circ}$ and $50^{\circ} \mathrm{S}$, derived from (a) MERRA and (b) the control integration. Positive values are shown with dashed contour lines. Negative values are shaded and shown with continuous contour lines.

drag) advances the wind reversal in the upper stratosphere by 14 days with respect to the control integration at $2 \mathrm{hPa}$, but still a delay of 11 days is found with respect to MERRA. The final warming date at $10 \mathrm{hPa}$ also shows an anticipation of 10 days with respect to the control integration and a delay of 7 days with respect to MERRA so that the weak launched momentum flux experiment reduces significantly the biases found in the control integration. As will be seen next, this improvement may be partially attributed to a more realistic EPFD with stronger westward forcing (indeed, it exceeds the forcing magnitude found in MERRA).

Figure 6 a shows the zonal mean gravity wave drag in the $80^{\circ}-50^{\circ} \mathrm{S}$ latitudinal band given by the integration with larger launched gravity wave momentum flux. As expected, increasing the launched gravity wave momentum flux leads to a stronger gravity wave drag compared to the control integration (Fig. 3c). A doubling of the launched momentum flux gives about a $65 \%$ increase in the gravity wave drag positive peak and up to a $22 \%$ increase in the negative peak. The positivenegative patterns in gravity wave drag are essentially equivalent to the control integration.

The changes in the strength of gravity wave drag bring about changes in the EPFD. Figure $6 \mathrm{~b}$ shows the EPFD in the experiment with stronger gravity wave drag. A weaker magnitude of EPFD is found in this experiment with respect to the control experiment until 30 days before the final warming date. The peak of EPFD in the control integration is $-6.2 \mathrm{~m} \mathrm{~s}^{-1} \mathrm{day}^{-1}$, while it is $-4.4 \mathrm{~m} \mathrm{~s}^{-1}$ day $^{-1}$ in the stronger launched gravity wave momentum flux experiment. Therefore, the changes in EPFD can be associated with an interaction mechanism between gravity wave drag and planetary waves; a stronger gravity wave drag triggers a weaker EPFD until day 30 before the final warming date. On the other hand, when gravity wave drag changes to eastward acceleration and therefore the gravity wave drag perturbation changes sign, the negative EPFD presents a slightly stronger magnitude in the increased launched momentum flux experiment, visible in the EPFD peak at $5 \mathrm{hPa}$ (it is $-3.5 \mathrm{~m} \mathrm{~s}^{-1} \mathrm{day}^{-1}$ in the control integration and $-4.06 \mathrm{~m} \mathrm{~s}^{-1}$ day $^{-1}$ in the experiment with increased launched gravity wave momentum flux). Also, the deceleration peak just after the final warming date at $10 \mathrm{hPa}$ is stronger in the experiment with increased launched gravity wave momentum flux. Therefore, there appears to be an interaction mechanism that tends to compensate the changes; a perturbation in gravity wave drag triggers the contrary response in EPFD. An explanation of this interaction mechanism is given in section $3 b$.

The gravity wave drag in the experiment with small launched gravity wave momentum flux (Fig. 6c) also has a similar temporal evolution to the gravity wave drag in the control integration and to the one in the large launched momentum flux integration. The gravity wave drag peaks are about 7 times smaller than the control integration. The evolution of EPFD (Fig. 6d) for this decreased launched gravity wave momentum flux experiment shows the highest resemblance with the one derived from MERRA, especially above $10 \mathrm{hPa}$. The 


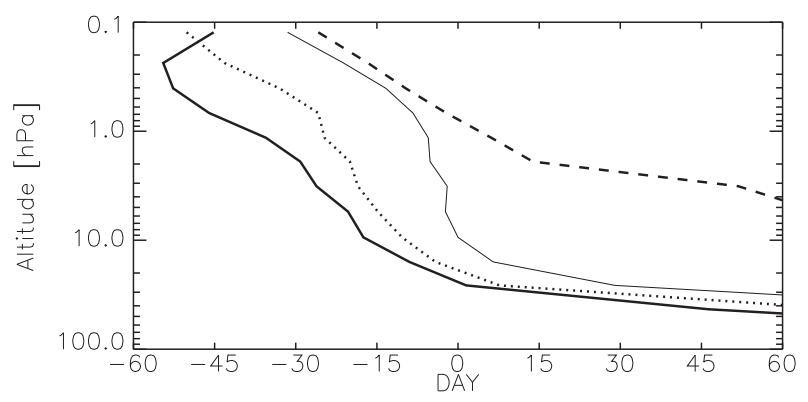

FIG. 5. Descent of the zero zonal mean zonal wind lines at $60^{\circ} \mathrm{S}$ in experiments with different launched gravity wave momentum flux: control integration (solid line), large launched gravity wave momentum flux experiment (dashed line), small launched gravity wave momentum flux experiment (dotted line), and MERRA (black thick line). The composites are conducted with respect to the control integration.

magnitudes of EPFD peaks are much stronger in this experiment, peaks of up to $-10.6 \mathrm{~m} \mathrm{~s}^{-1}$ day $^{-1}$ appear at $1 \mathrm{hPa}$ on day 42 prior to the final warming date. Closer to the final warming date, between day -15 and day +5 , when the sign in the gravity wave drag has changed and so has the sign of the perturbation in gravity wave drag, the EPFD is slightly weaker than the control experiment. Therefore, the EPFD response seems to be opposite to the unresolved gravity wave drag perturbation. In this experiment, again, we have found that the EPFD response through interactions between unresolved gravity waves and planetary waves tends to compensate the introduced gravity wave drag perturbation.

Figures $7 \mathrm{a}$ and $7 \mathrm{~b}$ show the gravity wave drag perturbation at $2 \mathrm{hPa}$ and at $10 \mathrm{hPa}$ introduced by the large launched momentum flux integration and the related response in EPFD. A smoothing of 10 days was applied in both sensitivity experiments to reduce the high variability of EPFD. Even when there is a high variability in the EPFD response (Fig. 7a shows that when the gravity wave drag perturbation is negative), the EPFD response tends to be positive. When the gravity wave drag perturbation changes sign, the EPFD response also shows a tendency to change sign. This negative EPFD response is more evident at $10 \mathrm{hPa}$ (Fig. $7 \mathrm{~b}$ ). At $2 \mathrm{hPa}$, there is a lag in the change of sign between EPFD response and gravity wave drag perturbation of about 20 days. Figure $7 \mathrm{c}$ shows the gravity wave perturbation and the response in EPFD for the small launched momentum flux experiment at $2 \mathrm{hPa}$; again, the EPFD response is opposite to the gravity wave drag perturbation. Both positive and negative EPFD responses are clearly visible in this experiment.

Following Cohen et al. (2013), the response of planetary wave drag to changes in gravity wave drag is measured with the scaled negative correlation of the changes of gravity wave drag and EPFD, the so-called degree of compensation (e.g., two completely anticorrelated time series will give a degree of compensation of 1). Figure $7 \mathrm{~d}$ shows the degree of compensation as a function of height. The degree of compensation is in both experiments greater than 0 for the whole height range, meaning (partial) canceling effects between the gravity wave drag perturbations and EPFD response. In the experiment with increased gravity wave drag, the largest interactions occur around $9 \mathrm{hPa}$, with a 0.43 degree of compensation. At $2 \mathrm{hPa}$, the degree of compensation is 0.06 (probably because of the lag between the two time series shown in Fig. 7a). Overall, an effect of partial cancellation is found along the middle stratosphere. Similarly the degree of compensation for the integration with reduced gravity wave drag also suggests that there is a compensation in the middle and upper stratosphere that maximizes around $3 \mathrm{hPa}$.

\section{b. Mechanism of interaction between gravity wave drag perturbations and EPFD responses}

Cohen et al. (2014) identified three possible mechanisms of interactions between gravity wave drag perturbations and the EPFD response. In the three mechanisms, the EPFD response tends to compensate the gravity wave drag perturbation consistently with the results we have found in the launched momentum flux sensitivity experiments shown in section 3a. The triggering of each mechanism depends on the latitudinal distribution of the potential vorticity. For the stability constraint mechanism, a weak latitudinal mean potential vorticity gradient is expected so that the perturbation in potential vorticity introduced by gravity wave drag may reverse locally the mean potential vorticity gradient; eventually, a sufficiently narrow and strong gravity wave drag perturbation may drive the stratosphere toward an unstable state even if the latitudinal mean potential vorticity gradient is large. The potential vorticity mixing constraint mechanism is expected to occur in the surf zone, where the potential vorticity is assumed to be uniform because of the efficient mixing produced by planetary wave breaking. The third mechanism involves changes in the planetary wave propagation produced by changes in the refraction index, which in turn are produced by the response of the zonal mean zonal wind to gravity wave drag perturbations. This is expected to work outside the surf zone close to its edge.

Figure 8 shows potential vorticity as a function of latitude at different heights during the vortex breakdown. The latitudinal potential vorticity distribution 90 days before the final warming is characterized by strong gradients; between 60 and 30 days before the final warming, there is a region around midlatitudes that has a 

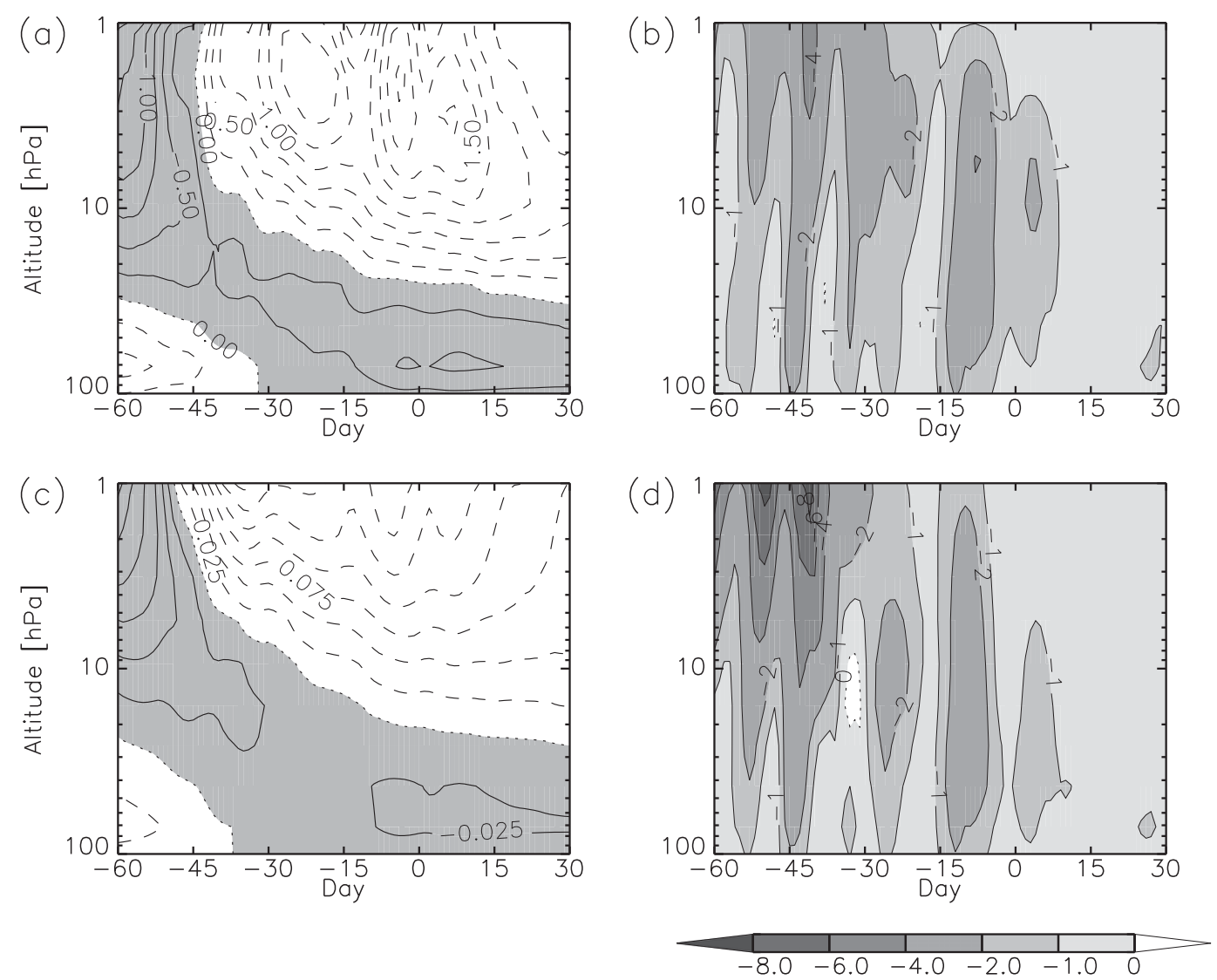

FIG. 6. (a) Zonal mean zonal gravity wave drag averaged between $80^{\circ}$ and $50^{\circ} \mathrm{S}$ from the integration with doubled launched gravity wave momentum flux (contour interval is $0.25 \mathrm{~m} \mathrm{~s}^{-1}$ day $^{-1}$; negative values are shaded). (b) Divergence of Eliassen-Palm flux for the same region and the same experiment. (c),(d) As in (a),(b), but for the integration with a reduced launched gravity wave momentum flux by 10 times (contour interval is $0.025 \mathrm{~m} \mathrm{~s}^{-1} \mathrm{day}^{-1}$ ). Positive values are shown with dashed contour lines. Negative values are shaded and shown with continuous contour lines.

decrease of potential vorticity gradient, which could be identified as a region of partial mixing, particularly at $10 \mathrm{hPa}$. At both heights, the range of latitudes between $80^{\circ}$ and $50^{\circ} \mathrm{S}$, where the compensation effects are examined, is characterized by large latitudinal potential vorticity gradients. The potential vorticity mixing constraint mechanism is unlikely to be present there. The gravity wave drag from the parameterization that has a steady and uniform launch spectrum is expected to be rather smooth temporally and latitudinally so that the stability constraint mechanism is not expected to be activated in this region of large latitudinal potential vorticity gradients. The mechanism that involves changes in the refraction index is, therefore, the only potential candidate.

The mechanism should involve zonal mean zonal wind perturbations established under stronger gravity wave drag conditions in the sensitivity experiments that diminish the index of refraction, so the propagation of planetary waves into the upper stratosphere diminishes. This situation, in turn, leads to a reduction of the Eliassen-Palm flux divergence associated with these planetary waves. To verify this hypothesis, the quasigeostrophic refractive index (Matsuno 1970) is calculated for stationary waves of wavenumber 1 as a reference. Figure 9a shows the dimensionless quasigeostrophic refractive index squared for the control integration. A large part of the examined $80^{\circ}-50^{\circ} \mathrm{S}$ latitudinal band lays on the waveguide of planetary wave propagation. The integration with increased launched gravity wave momentum flux produces a reduction of the index of refraction in the $80^{\circ}-50^{\circ} \mathrm{S}$ latitudinal band in the middle and lower stratosphere (Fig. 9b). Even larger differences are found in the upper stratosphere, resulting in an overall reduction of the efficiency for planetary wave propagation. The direct impact of the gravity wave drag in high latitudes is to directly diminish the potential vorticity gradient and, 

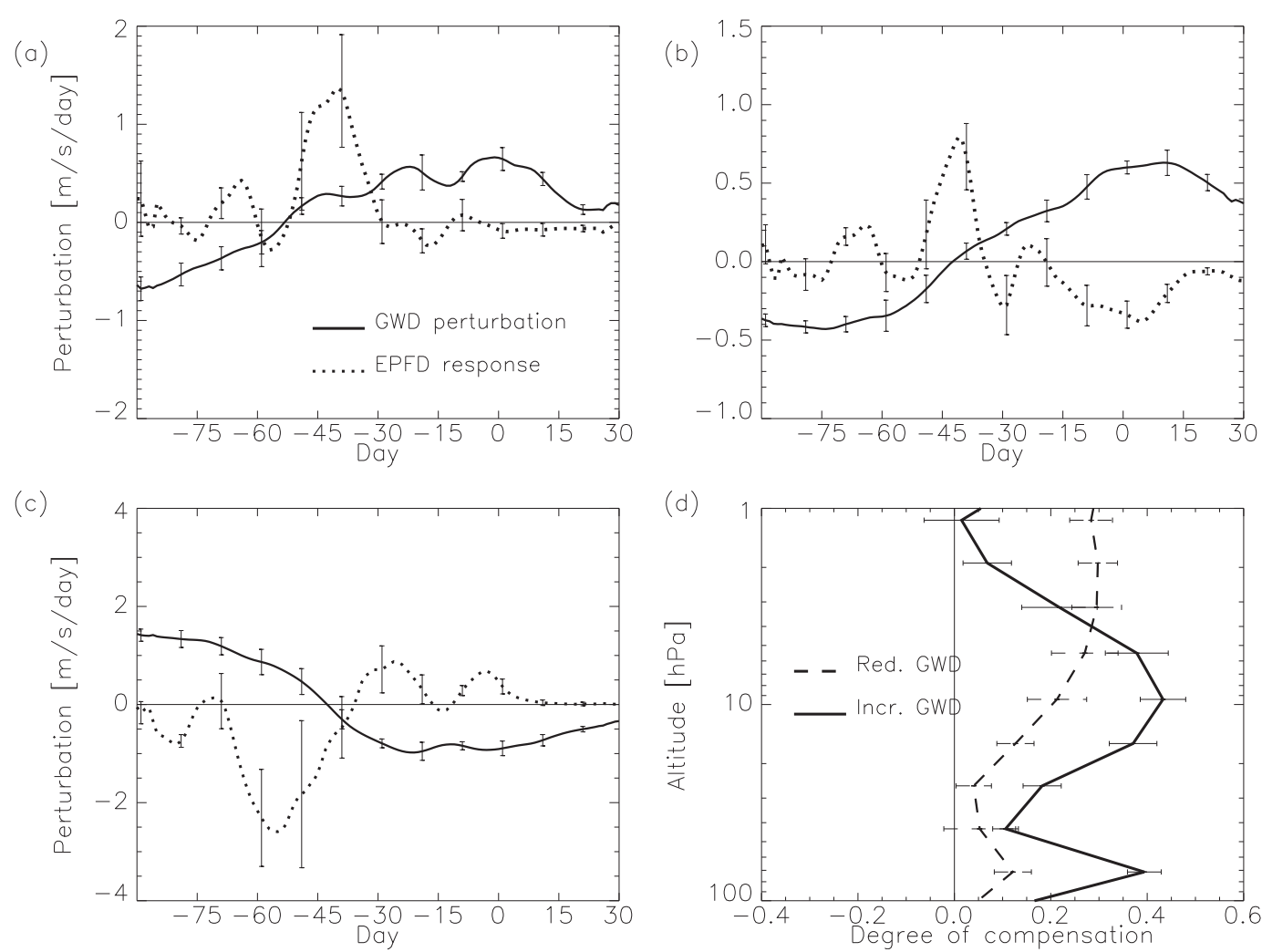

FIG. 7. Gravity wave drag perturbation (solid line) and the Eliassen-Palm flux divergence (dotted line) response and averaged between $80^{\circ}$ and $50^{\circ} \mathrm{S}$ (smoothed over 10 days). (a) Perturbation and response for the integration with doubled launched gravity wave momentum flux at $2 \mathrm{hPa}$. (b) As in (a), but at $10 \mathrm{hPa}$. (c) Perturbation and response for the integration with reduced launched gravity wave momentum flux at $2 \mathrm{hPa}$ and (d) degree of compensation as a function of height for the experiments with increased (continuous line) and decreased (dashed line) launched gravity wave momentum flux.

consequently, the refractive index (Cohen et al. 2014), so that the effect of an increased gravity wave drag in potential vorticity is an increase in the "effective mixing."

In contrast, the index of refraction for the weaker launched gravity wave momentum flux integration in the $80^{\circ}-50^{\circ} \mathrm{S}$ latitudinal band is larger than in the control experiment. Weaker zonal winds in middle latitudes and particularly changes from eastward to westward wind may induce a barrier for wave propagation [i.e., the critical surface (the zero zonal mean zonal wind surface) for quasi-steady planetary waves]. This barrier is found at higher latitudes in the experiment with weaker launched gravity wave momentum flux. Therefore, this barrier shrinks the waveguide, so the amplitude of upward-propagating planetary waves is increased. The response of planetary waves to the changes in the refractive index plays an instrumental role in the feedback processes that are tilting the critical surface and the polar vortex toward higher latitudes in height in the weaker launched gravity wave momentum flux experiment. Figures $10 \mathrm{a}-\mathrm{c}$ show the zonal mean zonal wind for the three experiments confirming this result.

The changes found in the Eliassen-Palm flux are consistent with the changes in the index of refraction. Figure 10a shows the Eliassen-Palm flux for the control integration. The Eliassen-Palm flux at $100 \mathrm{hPa}$ is strongest at $45^{\circ} \mathrm{S}$ (the zero wind critical surface is at about $27^{\circ} \mathrm{S}$ ). The strongest Eliassen-Palm flux is tilted toward higher latitudes as a function of height (following the jet tilt). At $1 \mathrm{hPa}$, the Eliassen-Palm flux is strongest at $58^{\circ} \mathrm{S}$. A weaker upward and equatorward Eliassen-Palm flux is found in the middle-to-upper stratosphere for the integration with the stronger launched gravity wave momentum flux (see Fig. 10b). In contrast, reducing gravity wave drag leads to more favorable conditions for upward propagation of planetary waves in high latitudes, as shown by the EliassenPalm flux difference vectors in the middle and upper stratosphere (Fig. 10c). Between $60^{\circ}$ and $40^{\circ} \mathrm{S}$ above $20 \mathrm{hPa}$, there is a mild decrease of Eliassen-Palm flux 
(a)

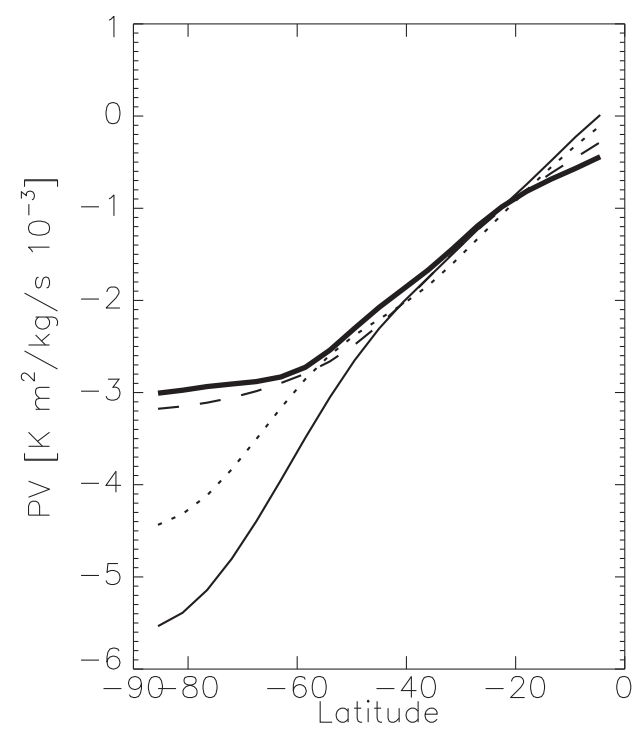

(b)

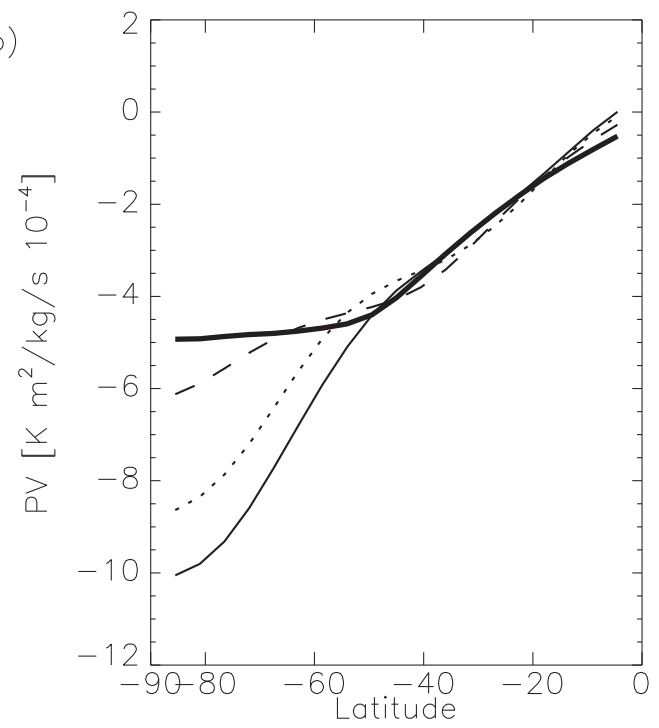

FIG. 8. Potential vorticity $\left(\mathrm{K} \mathrm{m}^{2} \mathrm{~kg}^{-1} \mathrm{~s}^{-1}\right)$ in the control integration as a function of latitude at (a) 2 and (b) $10 \mathrm{hPa}$ at different times: on the final warming date (continuous line), and 30 (dashed line), 60 (dotted line), and 90 days (dashed-dotted line) before the final warming date.

because of the presence of the barrier for propagation seen in Fig. 9c.

\section{c. Dependence of the stratospheric final warming on the strength of Eliassen-Palm flux}

Model integrations with an artificially increased and with a decreased bottom boundary Eliassen-Palm flux at $100 \mathrm{hPa}$ are examined here. The increased forced large-scale wave activity is expected to propagate upward in the model, increasing the Eliassen-Palm flux and therefore the forcing (EPFD) associated with these waves. Since these waves are providing the right forcing for the development of the vortex breakdown, we expect an earlier final warming in the model for the increased bottom boundary flux experiment and a later final warming for the decreased bottom boundary flux experiment.

Figure 11 shows the date of the wind reversal for the 7 -yr composite as a function of height. The integration with $25 \%$ increased planetary wave activity shows a slight improvement in the wind reversal date in the middle stratosphere with respect to the control integration, but the response is much weaker than when changing the launched momentum flux in the gravity wave drag parameterization. Regrettably, we are unable to perform experiments with stronger bottom boundary flux because dynamical instabilities arise in the model integration. In the experiment with $50 \%$ reduced planetary wave activity, a large difference is found with respect to the control integration, particularly in the middle and lower stratosphere. The final warming date in this integration shows a pronounced delay of more than 60 days with respect to the control integration. The wind reversal at $10 \mathrm{hPa}$ only occurs in 3 years, and it does not occur in the rest of the years. Below $15 \mathrm{hPa}$, the wind does not reverse for any of the analyzed years in the experiment with reduced bottom boundary flux.

Figure 12a shows the gravity wave drag evolution for the integration with reduced bottom boundary flux. Several differences should be noticed with respect to the control integration. First, the magnitude of the westward forcing peak found at $1 \mathrm{hPa}$ is larger than the one found in the control integration. The sensitivity of the temporal evolution of gravity wave drag in this experiment is much higher than the sensitivity found in the launch momentum flux experiments. The change from westward to eastward gravity wave drag occurs 15-20 days later than in the control integration, in coherence with the delay of the change of sign of the zonal wind vertical shear. At $10 \mathrm{hPa}$, the change in gravity wave drag from westward to eastward acceleration occurs 24 days before the final warming, while the change from positive vertical shear to negative shear in the lower stratosphere occurs 30 days before the final warming date (not shown). As expected, the reduction of the bottom boundary flux has a direct impact on the EPFD (Fig. 12b), with a reduction of the intensity of planetary wave drag. Two of the main peaks described in the previous section, 50 and 9 days before the final warming date, are attenuated. EPFD in the middle stratosphere is, in general, reduced between $50 \%$ and $75 \%$ up to the final warming date. 

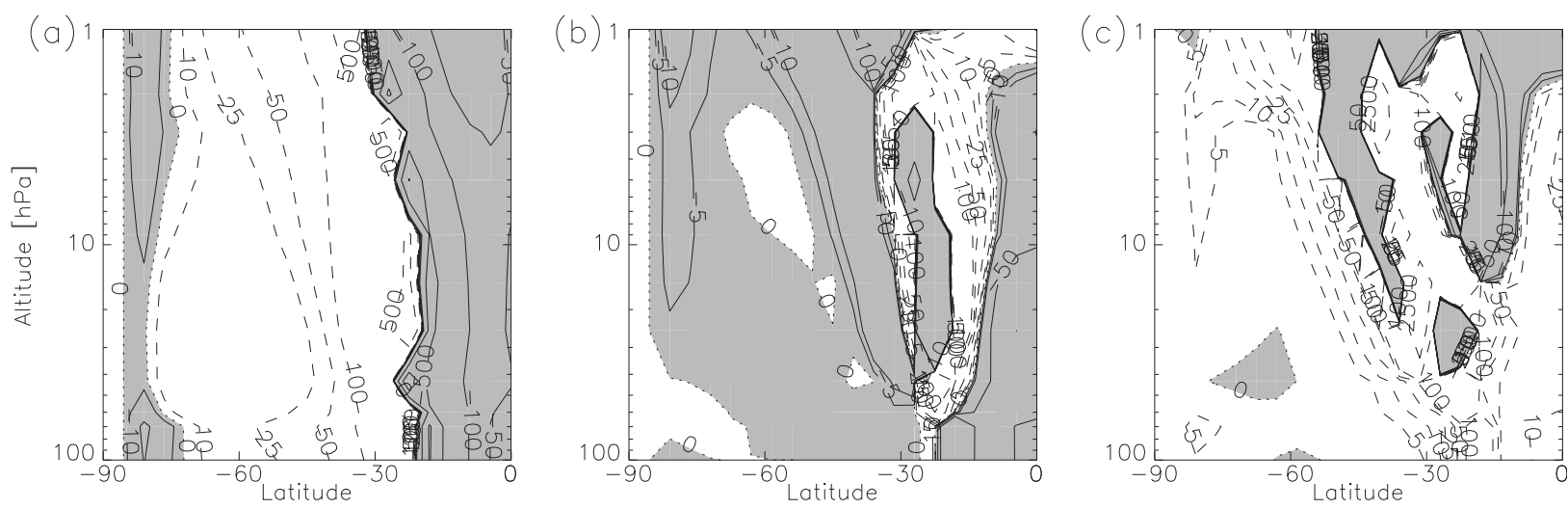

FIG. 9. Quasigeostrophic refractive index squared $n^{2}$ for zonal wavenumber 1 averaged between 45 and 15 days before the final warming date ( $n^{2}$ has been nondimensionalized by the square of Earth's radius). (a) Control experiment. (b) Differences between the index of refraction of the increased launched momentum flux integration and of the control integration. (c) Differences between the index of refraction of the reduced launched momentum flux integration and of the control integration. Contour intervals are in a logarithmic scale. Negative values are shaded.

In the experiment with increased bottom boundary flux, EPFD (Fig. 12d) shows a large resemblance with the control integration. A slight increase of EPFD on the order of $1 \mathrm{~m} \mathrm{~s}^{-1}$ day $^{-1}$ on average is found in the increased bottom boundary flux integration at the beginning of the wind reversal. The increase of bottom boundary flux also produces changes in gravity wave drag. The change of sign in the gravity wave drag at $1 \mathrm{hPa}$, from westward to eastward acceleration, shows an anticipation of 11 days with respect to the control integration (Fig. 12c). The change from eastward to westward acceleration at $100 \mathrm{hPa}$ also shows an anticipation of 11 days compared to the control integration and an anticipation of 29 days with respect to the decreased bottom boundary flux experiment. This is consistent with an earlier reversal of the zonal mean vertical shear. Therefore, the date of the change of sign in zonal wind shear is highly sensitive to the strength of bottom boundary flux; on the other hand, the change of sign in zonal wind presents a weaker sensitivity to the strength of the bottom boundary flux.

The experiment with a decreased bottom EliassenPalm flux has a stronger westward gravity wave drag from 60 to 30 days before the final warming date in the upper stratosphere, compared to the control integration, and a weaker eastward acceleration afterward. On the other hand, the experiment with an increased bottom Eliassen-Palm flux shows a weaker westward gravity wave drag. Figure 13 shows the perturbation introduced in EPFD and the response in gravity wave drag at $2 \mathrm{hPa}$. Gravity wave drag in the integration with reduced bottom Eliassen-Palm flux (Fig. 13a) shows a steady westward increase that seems to partially cancel the reduction of (westward) EPFD. The compensation is not total. There is a lag between the maximum EPFD perturbation and the minimum gravity wave drag response. A similar partial compensation effect occurs in the integration with increased bottom Eliassen-Palm flux (Fig. 13b). The westward EPFD perturbation leads to an eastward gravity wave drag response; the magnitude of the response is, on average, $20 \%$ smaller than the EPFD perturbation during the early stages of the vortex breakdown. The degree of compensation considering the perturbations to EPFD and the responses found in parameterized gravity wave drag for the two bottom Eliassen-Palm flux experiments is shown in Fig. 13c. The maximum cancellation is found at $1 \mathrm{hPa}$ (the degree of compensation is 0.5 and 0.38 for reduced and increased bottom Eliassen-Palm flux, respectively). The degree of compensation reverses at about $25 \mathrm{hPa}$. This countercompensation is explained in the next subsection.

\section{d. Mechanism of interaction between EPFD perturbations and gravity wave drag responses}

The experiments that vary the strength of the bottom Eliassen-Palm flux show that, when the EPFD is changed, the gravity wave drag also responds in the opposite sense. In other words, the gravity wave drag response from the parameterization tends to compensate the introduced EPFD perturbation.

Figure 14a shows the zonal mean zonal wind averaged between 45 and 15 days before the final warming date for the control integration, while Figs. 14b and 14c correspond to the case with reduced and increased bottom boundary Eliassen-Palm flux integrations, respectively. The reduction of bottom boundary flux, and 

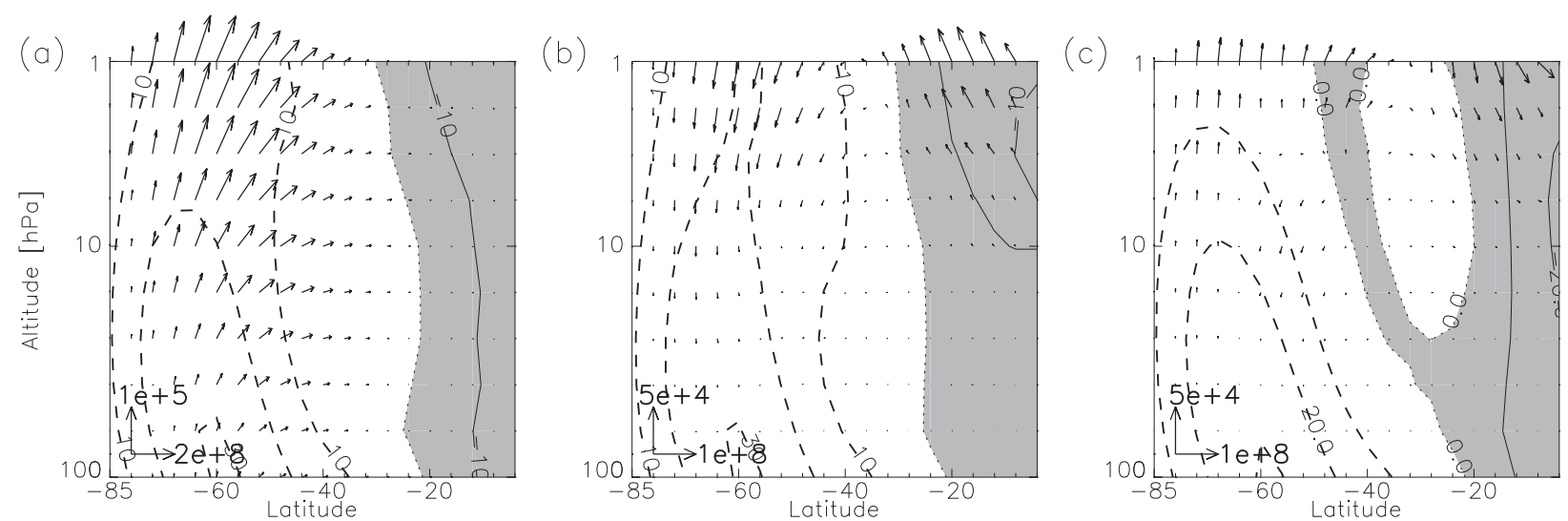

FIG. 10. (a) Zonal mean zonal wind (contours, $\mathrm{m} \mathrm{s}^{-1} \mathrm{day}^{-1}$ ) and Eliassen-Palm flux vectors $\left(\mathrm{kg} \mathrm{s}^{-2}\right.$, reference vector in lower-left corner) averaged between 45 and 15 days prior to final warming date for the control integration. (b) Zonal mean zonal wind for the integration with increased launched gravity wave momentum flux (contours) and differences between the Eliassen-Palm flux vectors for the integration with increased launched gravity wave momentum flux and for the control integration. (c) As in (b), but for the reduced launched gravity wave momentum flux integration. Negative values are shaded.

so EPFD, produces a strengthening of the winter polar jet above $25 \mathrm{hPa}$ and a generalized reduction in the tilt of the jet. The pronounced reduction of wind shear leads to a reduced parameterized gravity wave drag, as the parameterization relies on wind shear to deposit drag at each model level via critical-level filtering. In contrast, the wind shear in the lower stratosphere is larger for the increased bottom boundary flux integration (Fig. 14c) compared to the control integration, leading to a broader spectral range of westward intrinsic phase speed filtered in the lower stratosphere in the critical levels and, therefore, a larger westward gravity wave drag there. In the upper stratosphere, the eastward gravity wave drag that is produced because of wave saturation is consequently larger than the control integration, since the spectrum that propagates toward the upper stratosphere is more asymmetric. In other words, the extra part of the westward intrinsic phase speed range that was filtered in lower altitudes does not compensate the saturation of the corresponding eastward intrinsic phase speed waves at higher altitudes so that a larger eastward acceleration results in the experiment with increased bottom boundary flux.

To conclude, a stronger westward EPFD leads to a weaker polar jet and therefore a reduction of criticallevel filtering of eastward phase speed waves so that the acceleration produced by the net saturation of westward phase speed waves decreases. A weaker westward EPFD leads to the opposite response in gravity wave drag: a stronger polar jet and, thus, an increase of westward acceleration.

The interactions between EPFD changes and gravity wave drag responses are reminiscent of the gravity wave drag effects found in sudden stratospheric warmings, in which the stronger EPFD associated with the sudden stratospheric warming leads to changes in zonal winds that, in turn, result in a weaker gravity wave drag. The weakening in gravity wave drag was associated with a weakening of the meridional circulation, leading to a colder mesosphere (Holton 1983). Ren et al. (2008) also identified that abnormal planetary wave activity in a sudden stratospheric warming scenario leads to a weaker polar jet, which, in turn, affects the deposition of gravity wave momentum flux. The experiments shown in McLandress and McFarlane (1993) also appear to represent a compensation effect in the interactions between EPFD changes and orographic gravity wave drag responses; however, the interaction mechanism should be different because the orographic gravity waves are assumed to have a single

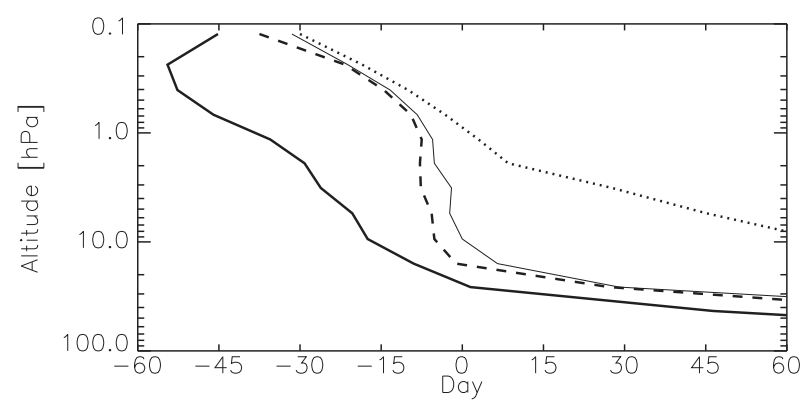

FIG. 11. Descent of the zero zonal mean zonal wind lines at $60^{\circ} \mathrm{S}$ in experiments with different bottom flux: increased bottom flux (dashed line), decreased bottom flux (dotted line), control integration (solid line), and MERRA (black thick line), using the control integration as reference for the composites. Positive values are shown with dashed contour lines. Negative values are shaded and shown with continuous contour lines. 

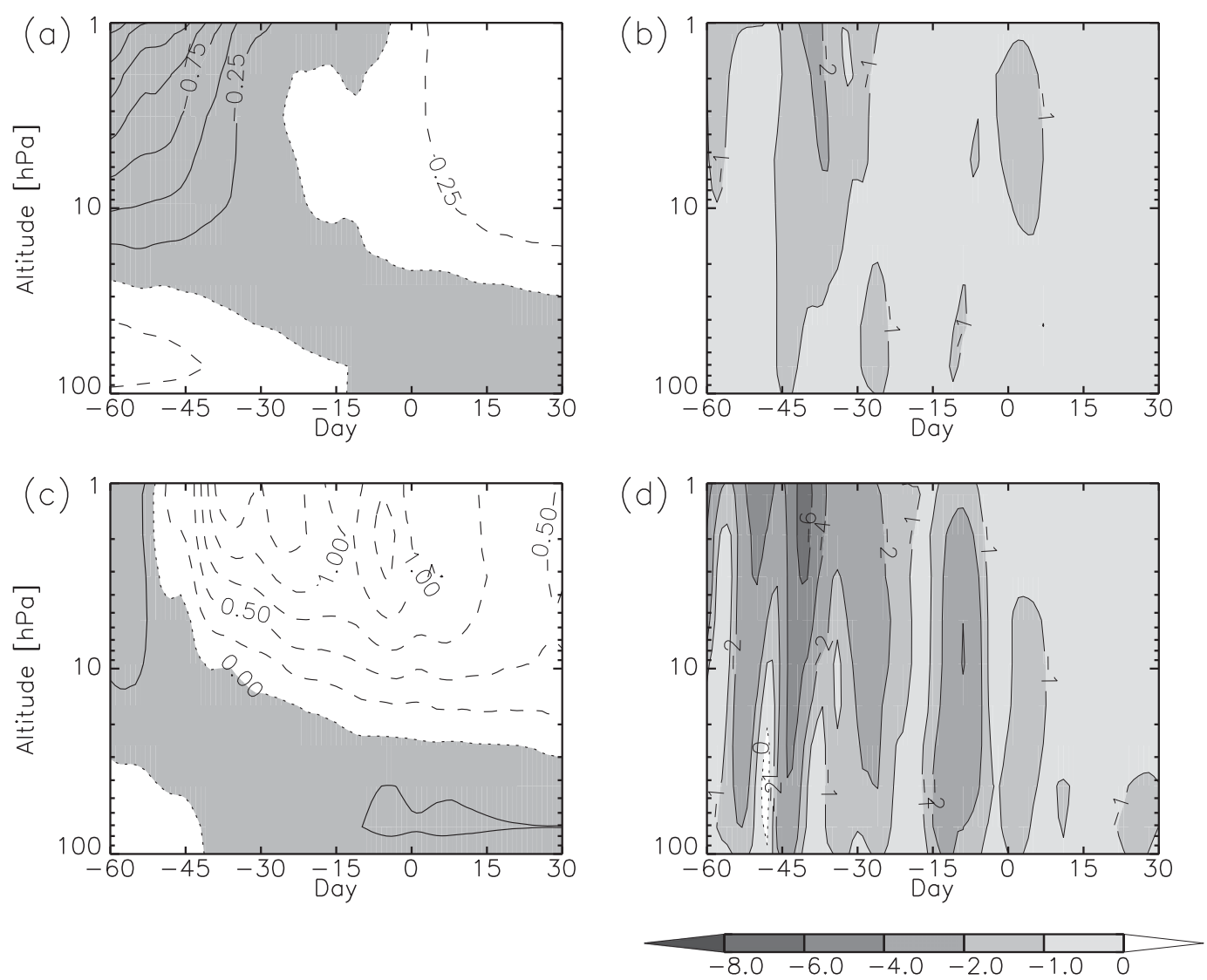

FIG. 12. (a) Zonal mean zonal gravity wave drag averaged between $50^{\circ}$ and $80^{\circ} \mathrm{S}$ from the gravity wave parameterization for the integration with $50 \%$ reduced bottom flux. (b) Divergence of Eliassen-Palm flux for the same region and the same integration. (c),(d) As in (a),(b), but for the integration with $25 \%$ increased bottom flux. Contour interval for (a),(c) is $0.25 \mathrm{~m} \mathrm{~s}^{-1}$ day $^{-1}$. Positive values are shown with dashed contour lines. Negative values are shaded and shown with continuous contour lines.

critical level (zero zonal wind), while the interaction mechanism that we describe needs a broad isotropic spectrum of (nonorographic) gravity waves, which results in multiple critical levels.

Below $25 \mathrm{hPa}$, the degree of compensation is negative (correlation positive; see Fig. 13c) because the response of gravity wave drag is dominated directly by critical-level filtering. A stronger planetary wave activity produces a stronger westward EPFD (negative EPFD perturbation); this diminishes the zonal wind shear, so the eastward gravity wave drag given by critical-level filtering is diminished. Therefore, a negative EPFD perturbation produces a negative gravity wave drag response; this gives a negative degree of compensation (positive correlation). This opposite response in the lower part of the vertical profile is related to the vertical dipole found in gravity wave drag profiles (see, for instance, Fig. 15), which is a consequence of gravity wave momentum flux conservation (Shepherd and Shaw 2004). Thus, countercompensation is inevitable in the gravity wave drag response to EPFD changes.

\section{Conclusions}

The impact of the interactions between planetary waves (resolved wave drag) and parameterized nonorographic gravity waves (unresolved wave drag) in the stratospheric final warmings of the Southern Hemisphere is examined through a middle-atmosphere model. Model results are compared with MERRA.

The increase of nonorographic gravity wave drag, via an increase of the launched gravity wave momentum flux of the parameterization, increases the delay of the stratospheric final warming with respect to observations. This degradation in model quality is attributable to changes in both resolved and parameterized wave drag. First, the filtering mechanism in the nonorographic parameterization leads to stronger eastward drag before the final warming date that alters the zonal mean flow 

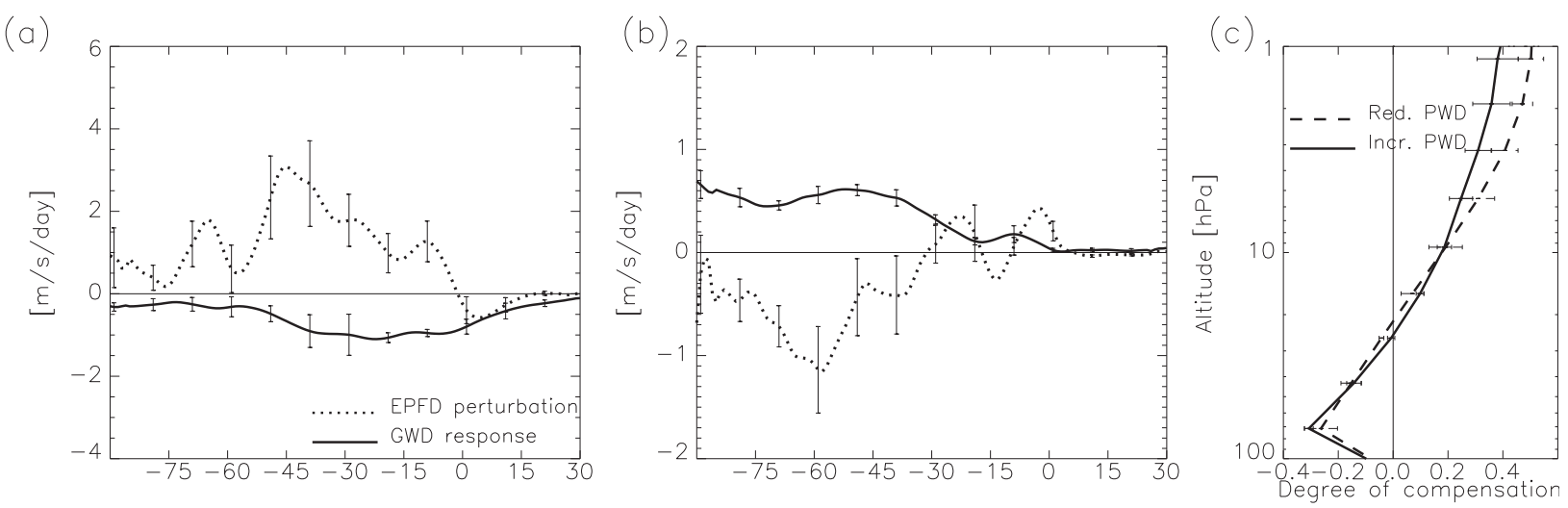

FIG. 13. EPFD perturbation (dotted line) and the zonal gravity wave drag response (continuous line) averaged between $80^{\circ}$ and $50^{\circ} \mathrm{S}$ at $2 \mathrm{hPa}$ (smoothed over 10 days). (a) Integration with reduced bottom Eliassen-Palm flux. (b) Integration with increased bottom EliassenPalm flux. (c) Degree of compensation for both increased and decreased Eliassen-Palm flux experiments.

during late spring. Then the changes in zonal mean circulation introduced by the changes in gravity wave forcing are, in turn, modifying the index of refraction for the propagation of planetary waves and so producing changes in the Eliassen-Palm flux divergence. This interaction mechanism produced by the response of the Eliassen-Palm flux divergence to perturbing the unresolved wave drag is in accordance with the one discussed previously in Cohen et al. (2014), Sigmond and Shepherd (2014), and Watson and Gray (2014). In the experiment with increase of launched gravity wave momentum flux, the Eliassen-Palm flux divergence diminishes significantly with respect to the control integration. In contrast, reducing the gravity wave momentum flux launched in the nonorographic parameterization leads to a stronger Eliassen-Palm flux divergence, which is closer to the one found in MERRA. This improves the representation of the springtime transition in the model integration.

By tuning the bottom boundary flux at $100 \mathrm{hPa}$, we were able to simulate scenarios with increased and decreased Eliassen-Palm flux divergence. In these scenarios, it was not possible to reach the same level of improvement in terms of the final warming date as in the integration with increased launched momentum flux, though it should be noted that, as a result of stability issues, it was not possible to increase the bottom boundary flux by more than $25 \%$. The only aspect that we changed from the bottom boundary flux is the intensity. Changing other aspects, such as the flux direction or the phase speed spectrum, may lead to a greater sensitivity of the vortex breakdown. One possible reason for the small impact of Eliassen-Palm flux changes to the final warming date may be the large compensation effect in the upper stratosphere produced by the gravity wave parameterization. About $40 \%$ of the introduced EPFD perturbation is compensated by gravity wave drag.

The explanation for this interaction mechanism of gravity wave drag to EPFD perturbations lays in the critical-level filtering mechanism in the parameterization. Changing the mean flow through an increased resolved Eliassen-Palm flux divergence leads to steeper vertical gradients of zonal wind that, in turn, filter a broader eastward phase speed range of the launched gravity wave spectrum in the parameterization. This produces an increase of eastward forcing in the lower stratosphere and an increase of westward forcing in the upper stratosphere. The change in the sign of the zonal acceleration given by the gravity wave parameterization during the vortex breakdown depends mainly on the change of sign in the vertical shear of zonal wind in the lower stratosphere. The change of sign in the vertical shear precedes by about 40 days the transition in the zonal wind at $10 \mathrm{hPa}$ so that the parameterization gives eastward acceleration during the final warming in the middle and upper stratosphere, while westward acceleration is needed in the model to drive an earlier zonal wind transition.

Therefore, the compensation in the interactions between gravity wave drag and planetary wave drag appears to be in both directions. Eliassen-Palm flux divergence responds to gravity wave drag perturbations by canceling them at least partially via changes in the index of refraction. Furthermore, gravity wave drag responds to Eliassen-Palm flux divergence perturbations by partially compensating them. These two compensating effects establish a feedback process between gravity wave drag and planetary wave drag. For instance, a reduction of westward gravity wave drag produces an increase of westward Eliassen-Palm flux divergence; this increase, in turn, produces a further reduction of 

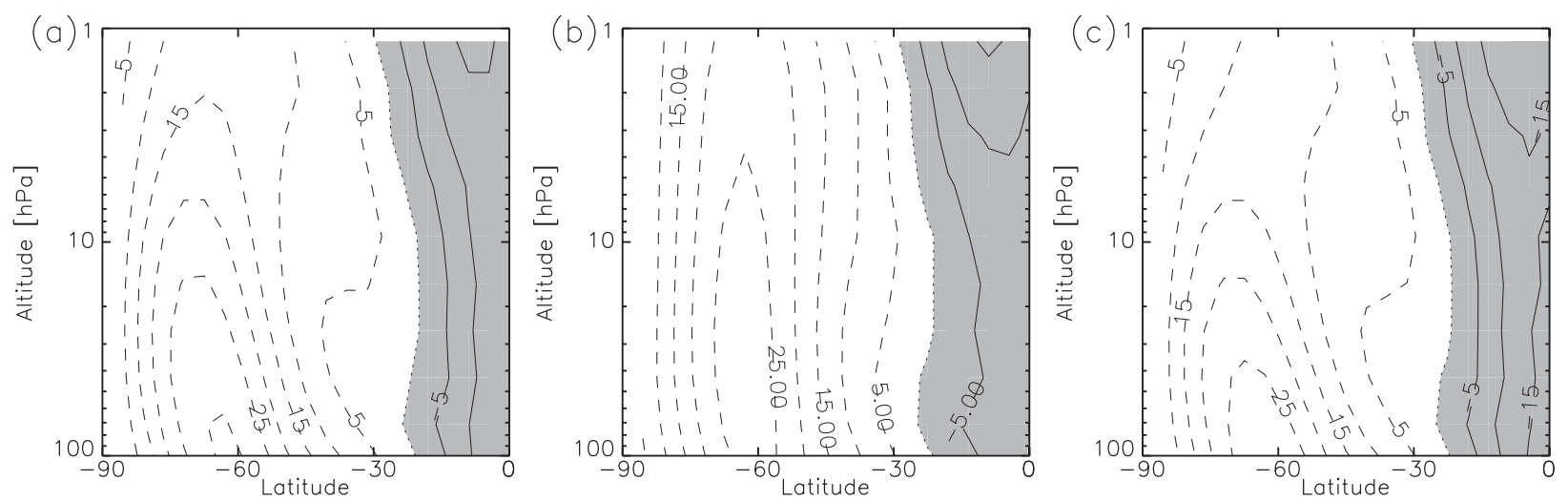

FIG. 14. Zonal mean zonal wind averaged between days 45 and 15 before the final warming date. (a) Control integration; (b) reduced bottom flux integration; and (c) increased bottom flux integration. Contour interval is $5 \mathrm{~m} \mathrm{~s}^{-1}$. Positive values are shown with dashed contour lines. Negative values are shaded and shown with continuous contour lines.

westward gravity wave drag. Gravity wave drag and planetary wave drag compensating interactions in the upper stratosphere are therefore expected to be robust and ubiquitous as a result of the feedback process. The interaction mechanism and the degree of compensation shown in this work are found for high latitudes in the Southern Hemisphere during winter-spring, where unresolved nonorographic gravity wave drag is expected to play a major role. The interaction mechanism and degree of compensation may not hold in other situations.

Our results show that Eliassen-Palm flux divergence has a dominant role in driving final warmings in the
Southern Hemisphere; however, the Eliassen-Palm flux divergence has a stronger sensitivity to the changes produced in the zonal mean conditions by gravity wave drag changes than to changes in the bottom EliassenPalm flux entering the stratosphere.

Orographic waves also play an important role in the stratospheric final warming because their phase speeds are close to zero, so the critical level will be close to the zonal wind zero surface. Because the model we use has a bottom boundary at $100 \mathrm{hPa}$, we cannot implement an orographic gravity wave parameterization in the model. These orographic parameterizations need the near-surface
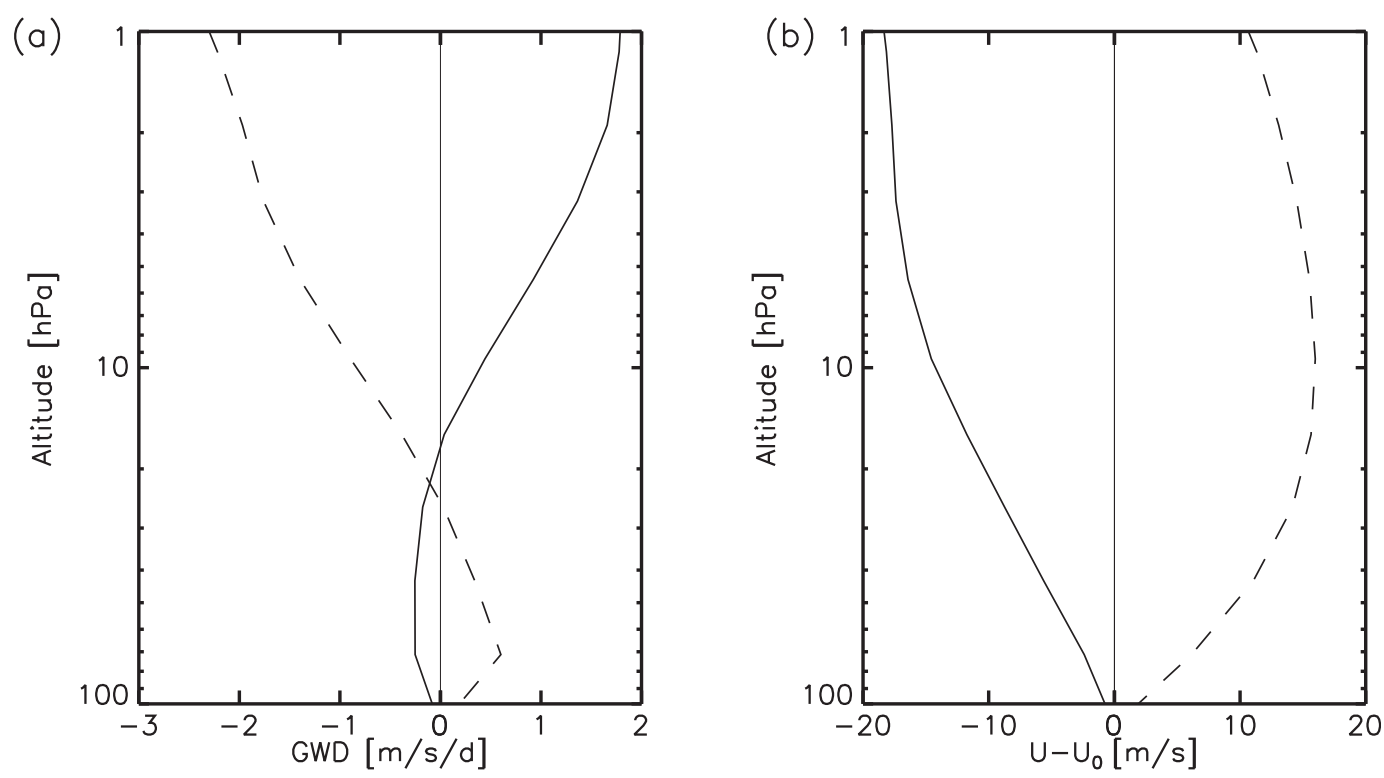

FIG. 15. (a) Zonal mean gravity wave drag vertical profiles from the nonorographic parameterization in the control experiment. (b) Intrinsic zonal mean zonal wind with respect to the launch height for the control integration ( $U_{0}$ is the zonal mean zonal wind at launch height). Solid lines correspond to vertical profiles at $60^{\circ} \mathrm{S}$ and 30 days before the final warming date. Dashed lines correspond to vertical profiles at $75^{\circ} \mathrm{S}$ and 60 days before the final warming date. 
and tropospheric winds to determine the parameterized orographic wave drag. Because of this, we were unable to evaluate the role of orographic gravity waves with sensitivity experiments such as the ones conducted for planetary and orographic waves by McLandress et al. (2012) and Sigmond and Shepherd (2014). The interaction mechanism (EPFD changes gravity wave drag response as explained in the present work) is not expected to hold for orographic gravity wave drag parameterizations, since orographic waves are assumed to have a single frequency $(\omega=0)$ and, thus, a single critical level, while the mechanism described here needs a broad phase speed spectrum, which in turn leads to multiple critical levels.

As shown in this work, the tuning of gravity wave drag parameterizations focused on model biases, as, for instance, a delay in the vortex breakdown with respect to observations may lead to unexpected responses because of the current evidence of strong compensation between resolved and unresolved gravity wave drag. On the other hand, the estimation of parameters with data assimilation, such as four-dimensional variational assimilation (Pulido and Thuburn 2008; Pulido et al. 2012) or an ensemble Kalman filter (Ruiz et al. 2013), is expected to account for feedback processes in the model, giving an optimal configuration. A follow-up work will focus on the optimization of the parameterization using these assimilation techniques.

Acknowledgments. This work was partially funded by an Agencia Nacional de Promoción Científica y Tecnológica Grant PICT 2011-2452 and a CONICET Grant PIP 112-20120100414CO. This work was performed using computational facilities at CECONEA (UNNE). We thank the Editor, Rolando Garcia, Naftali Cohen, and an anonymous reviewer for insightful comments that helped to improve the manuscript. This work was, in part, motivated by an ISSI gravity wave team workshop.

\section{APPENDIX}

\section{Critical-Level Filtering in the Spectral Gravity Wave Parameterization}

As shown in the experiments of section 3a, parameterized zonal gravity wave drag shows a change of sign from westward to eastward acceleration starting at $1 \mathrm{hPa}$ at around day 45 before the final warming date and descending with time (see Figs. $3 c$ and 6a,c). Two characteristic mean gravity wave drag vertical profiles in the $80^{\circ}-50^{\circ} \mathrm{S}$ latitudinal band are apparent during the final warming. First a vertical dipole with negative (westward) acceleration above $30 \mathrm{hPa}$ and positive (eastward) acceleration below that remains up to day -45 , and then it switches to the inverse dipole: positive acceleration above and negative below. The dipolar structure is the consequence of momentum flux conservation in the parameterization (Shepherd and Shaw 2004).

We examine the filtering and saturation mechanisms in the spectral nonorographic gravity wave drag parameterization that lead to the change from westward to eastward acceleration in the parameterization. The gravity wave drag field as a function of latitude and height shows that the two dominant dipolar patterns are found at $75^{\circ} \mathrm{S}$ for negative acceleration aloft and positive below and at $60^{\circ} \mathrm{S}$ for the inverse dipole (not shown). Figure $15 \mathrm{a}$ shows the gravity wave drag profiles at $75^{\circ} \mathrm{S}$ and 60 days before the final warming date and at $60^{\circ} \mathrm{S}$ and 30 days before the final warming date for the control integration. The dipolar patterns with opposite behavior as a function of height are clearly visible. Figure $15 \mathrm{~b}$ shows the intrinsic zonal mean zonal wind to the launch height of the gravity waves in the parameterization. The (ground based) zonal wind at the launch height is about $29 \mathrm{~m} \mathrm{~s}^{-1}$ at both situations; however, this has no role in the propagation of the gravity waves in the parameterization. The zonal wind shear changes its sign during an earlier stage of the vortex breakdown than the transition from westerlies to easterlies, as seen for the intrinsic zonal mean zonal wind profile 30 days before the final warming date (continuous line in Fig. 15b).

An isotropic intrinsic gravity wave spectrum is propagated upward from the launch height (at around $100 \mathrm{hPa}$ ) by the parameterization. On day -60 the waves with positive (eastward) phase speed between 0 and $15 \mathrm{~m} \mathrm{~s}^{-1}$ are filtered since they encounter critical levels at the height range between 100 and $10 \mathrm{hPa}$ (dashed line in Fig. 15b); this critical-level filtering produces eastward forcing in the lower stratosphere (dashed line in Fig. 15a). In the upper part, the zonal wind does not vary on height practically. The spectrum of waves becomes saturated at those altitudes. Since the gravity wave spectrum is mainly dominated by westward intrinsic phase speed waves, a westward forcing results in the upper stratosphere.

The inverse situation is present on day -30 . Waves with westward intrinsic phase speed are filtered in the lower stratosphere so that a positive-negative gravity wave drag dipole results (continuous line in Fig. 15a). Note that, by this reasoning, the height of the change of sign in the gravity wave drag profile is given entirely by the depth of the shear layer in the lower stratosphere. To conclude, because of the filtering mechanism in an intrinsic isotropic gravity wave spectrum, the change from westward to eastward acceleration in 
the parameterization is produced when the zonal wind changes from positive to negative shear in the lowmiddle stratosphere.

Manzini and McFarlane (1998) found sensitivity to the launch height of the spectrum. The winter polar stratosphere in the Southern Hemisphere was improved when the gravity waves were launched from the surface in the parameterization. Regrettably, the launching height of gravity waves cannot be changed to the surface in our model, because the bottom boundary is at the tropopause height. Furthermore, orographic gravity waves are also expected to have important effects close to the height of the transition from westerlies to easterlies; however, we are also unable to represent them in this middle-atmosphere model.

\section{REFERENCES}

Andrews, D. G., J. R. Holton, and C. B. Leovy, 1987: Middle Atmosphere Dynamics. International Geophysics Series, Vol. 40, Academic Press, 489 pp.

Austin, J., and Coauthors, 2003: Uncertainties and assessments of chemistry-climate models of the stratosphere. Atmos. Chem. Phys., 3, 1-27, doi:10.5194/acp-3-1-2003.

Baldwin, M. P., D. B. Stephenson, D. W. Thompson, T. J. Dunkerton, A. J. Charlton, and A. O'Neill, 2003: Stratospheric memory and skill of extended-range weather forecasts. Science, 301, 636-640, doi:10.1126/science.1087143.

Black, R. X., and B. A. McDaniel, 2007: Interannual variability in the Southern Hemisphere circulation organized by stratospheric final warming events. J. Atmos. Sci., 64, 2968-2974, doi:10.1175/JAS3979.1.

Bloom, S. C., L. L. Takacs, A. M. Da Silva, and D. Ledvina, 1996: Data assimilation using incremental analysis updates. Mon. Wea. Rev., 124, 1256-1271, doi:10.1175/ 1520-0493(1996)124<1256:DAUIAU>2.0.CO;2.

Butchart, N., and Coauthors, 2011: Multimodel climate and variability of the stratosphere. J. Geophys. Res., 116, D05102, doi:10.1029/2010JD014995.

Cohen, N. Y., E. P. Gerber, and O. Bühler, 2013: Compensation between resolved and unresolved wave driving in the stratosphere: Implications for downward control. J. Atmos. Sci., 70, 3780-3798, doi:10.1175/JAS-D-12-0346.1.

,-- , and,- 2014 : What drives the Brewer-Dobson circulation? J. Atmos. Sci., 71, 3837-3855, doi:10.1175/JAS-D-14-0021.1.

Eyring, V., and Coauthors, 2006: Assessment of temperature, trace species, and ozone in chemistry-climate model simulations of the recent past. J. Geophys. Res., 111, D22308, doi:10.1029/ 2006JD007327.

Garfinkel, C. I., L. D. Oman, E. A. Barnes, D. W. Waugh, M. H. Hurwitz, and A. M. Molod, 2013: Connections between the spring breakup of the Southern Hemisphere polar vortex, stationary waves, and air-sea roughness. J. Atmos. Sci., 70, 2137-2151, doi:10.1175/JAS-D-12-0242.1.

Gerber, E. P., and Coauthors, 2010: Stratosphere-troposphere coupling and annular mode variability in chemistry-climate models. J. Geophys. Res., 115, D00M06, doi:10.1029/ 2009JD013770.

Global Modeling and Assimilation Office, 2011: MERRA 3D analyzed state, meteorology instantaneous 6-hourly, version
5.2.0. NASA Goddard Space Flight Center Distributed Active Archive Center, accessed May 2013, doi:10.5067/ ADAWH64DCRU0.

Holton, J. R., 1983: The influence of gravity wave breaking on the general circulation of the middle atmosphere. J. Atmos. Sci., 40, 2497-2507, doi:10.1175/1520-0469(1983)040<2497: TIOGWB $>2.0 . \mathrm{CO} ; 2$.

Hurwitz, M. M., P. A. Newman, F. Li, L. D. Oman, O. Morgenstern, P. Braesicke, and J. A. Pyle, 2010: Assessment of the breakup of the Antarctic polar vortex in two new chemistry-climate models. J. Geophys. Res., 115, D07105, doi:10.1029/2009JD012788.

Manzini, E., and N. A. McFarlane, 1998: The effect of varying the source spectrum of a gravity wave parameterization in a middle atmosphere general circulation model. J. Geophys. Res., 103, 31 523-31 539, doi:10.1029/98JD02274.

Matsuno, T., 1970: Vertical propagation of stationary planetary waves in the winter Northern Hemisphere. J. Atmos. Sci., 27, 871-883, doi:10.1175/1520-0469(1970)027<0871: VPOSPW $>2.0 . \mathrm{CO} ; 2$.

McLandress, C., and N. A. McFarlane, 1993: Interactions between orographic gravity wave drag and forced stationary planetary waves in the winter Northern Hemisphere middle atmosphere. J. Atmos. Sci., 50, 1966-1990, doi:10.1175/ 1520-0469(1993)050<1966:IBOGWD>2.0.CO;2.

— , T. G. Shepherd, S. Polavarapu, and S. R. Beagley, 2012: Is missing orographic gravity wave drag near $60^{\circ} \mathrm{S}$ the cause of the stratospheric zonal wind biases in chemistry-climate models? J. Atmos. Sci., 69, 802-818, doi:10.1175/ JAS-D-11-0159.1.

Pulido, M., and J. Thuburn, 2005: Gravity wave drag estimation from global analyses using variational data assimilation principles. I: Theory and implementation. Quart. J. Roy. Meteor. Soc., 131, 1821-1840, doi:10.1256/qj.04.116.

— and - 2008: The seasonal cycle of gravity wave drag in the middle atmosphere. J. Climate, 21, 4664-4679, doi:10.1175/ 2008JCLI2006.1.

_ S. Polavarapu, T. Shepherd, and J. Thuburn, 2012: Estimation of optimal gravity wave parameters for climate models using data assimilation. Quart. J. Roy. Meteor. Soc., 138, 298-309, doi:10.1002/qj.932.

Ren, S., S. Polavarapu, and T. Shepherd, 2008: Vertical propagation of information in a middle atmosphere data assimilation system by gravity-wave drag feedbacks. Geophys. Res. Lett., 35, L06804, doi:10.1029/2007GL032699.

Rienecker, M. M., and Coauthors, 2011: MERRA: NASA's Modern-Era Retrospective Analysis for Research and Applications. J. Climate, 24, 3624-3648, doi:10.1175/ JCLI-D-11-00015.1.

Ruiz, J., M. Pulido, and T. Miyoshi, 2013: Estimating model parameters with ensemble-based data assimilation. A review. J. Meteor. Soc. Japan, 91, 79-99, doi:10.2151/jmsj.2013-201.

Scinocca, J. F., 2003: An accurate spectral nonorographic gravity wave drag parameterization for general circulation models. J. Atmos. Sci. $\quad$ 60, 667-682, doi:10.1175/1520-0469(2003)060<0667: AASNGW $>2.0 . \mathrm{CO} ; 2$.

Shepherd, T. G., and T. A. Shaw, 2004: The angular momentum constraint on climate sensitivity and downward influence in the middle atmosphere. J. Atmos. Sci., 61, 2899-2908, doi:10.1175/JAS-3295.1.

Shine, K., 1987: The middle atmosphere in the absence of dynamical heat fluxes. Quart. J. Roy. Meteor. Soc., 113, 603-633, doi:10.1002/qj.49711347610. 
Sigmond, M., and T. Shepherd, 2014: Compensation between resolved wave driving and parameterized orographic gravity wave driving of the Brewer-Dobson circulation and its response to climate change. J. Climate, 27, 5601-5610, doi:10.1175/JCLI-D-13-00644.1.

Simpson, I. R., P. Hitchcock, T. G. Shepherd, and J. F. Scinocca, 2011: Stratospheric variability and tropospheric annular-mode timescales. Geophys. Res. Lett., 38, L20806, doi:10.1029/ 2011 GL049304.

Stolarski, R. S., A. R. Douglass, M. Gupta, P. A. Newman, S. Pawson, M. R. Schoeberl, and J. E. Nielsen, 2006: An ozone increase in the Antarctic summer stratosphere: A dynamical response to the ozone hole. Geophys. Res. Lett., 33, L21805, doi:10.1029/2006GL026820.

Thuburn, J., 1997: A PV-based shallow-water model on a hexagonal-icosahedral grid. Mon. Wea. Rev., 125, 2328-2347, doi:10.1175/1520-0493(1997)125<2328:APBSWM>2.0.CO;2.

Watson, P. A. G., and L. J. Gray, 2014: The stratospheric wintertime response to applied extratropical torques and its relationship with the annular mode. Climate Dyn., 44, 25132537, doi:10.1007/s00382-014-2359-2. 